\title{
Behavioral Foundations of Nested Stochastic Choice and Nested Logit*
}

\author{
Matthew Kovach Gerelt Tserenjigmid
}

February 7, 2022

\begin{abstract}
We provide the first behavioral characterization of nested logit, a foundational and widely applied discrete choice model, through the introduction of a non-parametric version of nested logit that we call Nested Stochastic Choice (NSC). NSC is characterized by a single axiom that weakens Independence of Irrelevant Alternatives based on revealed similarity to allow for the similarity effect. Nested logit is characterized by an additional menu-independence axiom. Our axiomatic characterization leads to a practical, data-driven algorithm that identifies the true nest structure from choice data. We also discuss limitations of generalizing nested logit by studying the testable implications of cross-nested logit.
\end{abstract}

Keywords: Nested Logit; Nested Stochastic Choice; Luce Model; IIA; Similarity Effect; Regularity; Revealed Similarity; Cross-Nested Logit; Nest Identification.

JEL: D01, D81, D9.

\section{Introduction}

Nested logit (Ben-Akiva (1973), McFadden (1978)) is the most widely applied generalization of multinomial logit (or Luce's (1959) model) due to its ability to capture various substitution patterns. ${ }^{1}$ In nested logit, each alternative belongs to a nest (or subset) of "similar" alternatives, and choice may be decomposed into two Luce procedures: the probability that $a$ is chosen from

\footnotetext{
*Kovach: Virginia Tech (mkovach@vt.edu); Tserenjigmid: UC Santa Cruz (gtserenj@ucsc.edu ). We thank José Apesteguia, Miguel A. Ballester, Khai Chiong, Ian Crawford, Federico Echenique, Mira Frick, Sean Horan, Ryota Iijima, Shaowei Ke, Jay Lu, Fabio Maccheroni, A.A.J. Marley, Paulo Natenzon, Pietro Ortoleva, Collin Raymond, Matthew Shum, Tomasz Strzalecki, the audiences of the BGSE Summer Forum 2018 and SAET 2019, and the seminar participants at UC Santa Cruz, University of Sussex, University of Toronto, KAIST, and Hitotsubashi University. We are also very grateful to the editor, Emir Kamenica, and four anonymous referees for their excellent suggestions and feedback.

${ }^{1}$ Nested logit has been used to study transportation demand (Anderson and De Palma (1992), Forinash and Koppelman (1993)), airline competition (Lurkin, Garrow, Higgins, Newman, and Schyns (2018)), automobile demand (Brownstone and Small (1989), Goldberg (1995)), telephone use (Train, McFadden, and Ben-Akiva (1987), Lee (1999)), and much more. See Chapter 4 of Train (2009) for an excellent discussion.
} 
menu $A$ is the probability that $a$ 's nest is chosen from among nests available in $A$ multiplied by the conditional probability that $a$ is chosen from that nest. Despite its immense importance, nested logit has escaped behavioral characterization. In this paper, we provide the first behavioral characterization of nested logit through the introduction of a fully non-parametric version that we term Nested Stochastic Choice (NSC). This axiomatic characterization sheds light on the implicit assumptions behind nested logit and related models and leads to a tractable method to identify (unobserved) nests from data.

Nested logit was developed to address the limitations of multinomial logit when dealing with "similar alternatives." In the Luce model, choice probabilities are proportional to a utility index and hence satisfy Independence of Irrelevant Alternatives (IIA): probability ratios are menuindependent. The similarity effect (Debreu (1960), Tversky (1972)) is a violation of IIA in which the introduction of an alternative to a menu has a much larger effect on the choice probabilities of alternatives of a "similar type" than on those of a "different type." Nested logit allows the similarity effect by assuming nested (similar) alternatives are more substitutable (e.g., because they receive a correlated utility shock). Our main result shows that nesting of similar alternatives, a key behavioral feature of nested logit (and also NSC), is captured by a weakening of IIA that allows for the similarity effect. This finding reveals a deep connection between nested logit and the similarity effect.

To illustrate the similarity effect, consider the red bus/blue bus example from Debreu (1960). A commuter making a choice between a red bus and a train may choose either with probability 0.5. If the option to take a blue bus is introduced, it is plausible that it will have no effect on the commuter's likelihood of taking the train; the blue bus only affects the probability of selecting the red bus (e.g., by reducing it to 0.25 ). The intuition behind this is that the buses are similar to each other in a way that neither one is to the train. Nested logit handles this by placing the two buses into a bus nest. While this example provides an extreme case of the similarity effect (the buses are perfect substitutes, or "duplicates"), the principle that "similar alternatives affect each other" readily extends to many situations of interest to economists, firms, and policy-makers, such as a consumer's choice of vehicle or apartment. ${ }^{2}$

Our analysis of nested logit relies on the introduction of NSC, a non-parametric version of nested logit. Formally, a stochastic choice function $p$ is an NSC if there exist nests $X_{1}, \ldots, X_{K}$ that partition the set of all alternatives $X$ and functions $v$ and $u$ such that, for each choice set $A$, the probability of choosing $a \in A \cap X_{i}$ is given by

$$
p(a, A)=\frac{v\left(A \cap X_{i}\right)}{\sum_{j=1}^{K} v\left(A \cap X_{j}\right)} \frac{u(a)}{\sum_{b \in A \cap X_{i}} u(b)} .
$$

The NSC is defined by two Luce rules where the attractiveness of the nest $A \cap X_{i}$ is measured by $v\left(A \cap X_{i}\right)$, and the attractiveness of the alternative $a$ is measured by $u(a)$ (or simply Luce's utility

\footnotetext{
${ }^{2}$ In these cases, the correct nest specification is not easily observed by the analyst. For instance, apartments in a city might be nested based on subjective neighborhoods, which may depend on a variety of factors. In turn, some of these factors may be observable while others may be subjective or difficult to observe.
} 
of $a$ ). In terms of the red bus/blue bus example, $v$ governs the choice between general modes, "a bus" or "a train," while $u$ governs the choice between specific alternatives, the red or blue bus.

Notice that nested logit is the special case of NSC in which, for each $i \leq K$,

$$
v\left(A \cap X_{i}\right)=\left(\sum_{a \in A \cap X_{i}} u(a)\right)^{\eta_{i}} \text { for some } \eta_{i}>0 .
$$

Hence, NSC is nested logit without any assumptions on the relationship between $v$ and $u$. It is commonly assumed in the applied literature that $\eta_{i} \leq 1$, as this ensures that nested logit is a Generalized Extreme Value (GEV) model (McFadden, 1978), and therefore this restriction is sufficient for consistency with the random utility model (RUM). However, this restriction $\left(\eta_{i} \leq 1\right)$ is not necessary for Equation 2 to be a RUM and nested logit has been estimated without this restriction. ${ }^{3}$ Therefore, for simplicity of exposition we will refer to Equation 2 as a nested logit for any parameter value. To provide further clarity, we may sometimes refer to nested logit satisfying the restriction $\left(\eta_{i} \leq 1\right)$ as the random utility (RU) nested logit. We provide behavioral foundations for NSC and nested logit, along with a characterization of random utility nested logit.

We utilize a revealed preference approach to identify the subjective/endogenous nest structure of the NSC. This is achieved by introducing a notion of revealed similarity. In nested logit, if two alternatives are in the same nest, then their probability ratio will always be independent of other alternatives. Consistent with nested logit, we use this insight to define a notion of similarity: alternatives $a$ and $b$ are revealed categorically similar, denoted $a \sim_{p} b$, if IIA holds between $a$ and $b$ at any menu. Otherwise, they are revealed categorically dissimilar. Therefore, the core notion of similarity underpinning nested logit is binary: alternatives are similar or not.

Equipped with this notion of reveled similarity, we can weaken IIA to allow for the similarity effect. To do so, we decompose IIA into two axioms. The first axiom, Independence of Symmetric Alternatives (ISA), imposes an IIA condition between $a$ and $b$ in the presence of a third alternative $x$, when $x$ is symmetrically related to $a$ and $b$ in terms of revealed similarity (i.e., both are revealed categorically similar or dissimilar to $x$ ). In terms of the red bus/blue bus example, IIA should hold between the buses in the presence of the train. The second axiom, Independence of Asymmetric Alternatives (IAA), "completes" ISA by imposing an IIA condition when $x$ is asymmetrically similar to $a$ and $b$ (i.e., $x$ is similar to one and not the other). For example, IAA implies that the introduction of the blue bus impacts the red bus and the train equally, which directly rules out the similarity effect.

Our main result is that ISA characterizes NSC (Theorem 1). Further, since ISA is the minimal

\footnotetext{
${ }^{3}$ This parameter restriction is sometimes referred to as the Daly-Zachary-McFadden condition (Daly and Zachary (1978), McFadden (1978)), as they showed that this is sufficient for consistency with RUM for arbitrary values of the other variables (e.g., utilities). However, this restriction is not always imposed. For instance, Train, McFadden, and Ben-Akiva (1987) provide estimates of a model for which $\eta_{i}>1$, remarking that it represents greater substitutability between nests than within nests (see also Train, Ben-Akiva, and Atherton (1989), Lee (1999), Foubert and Gijsbrechts (2007)). Further, nested logit with $\eta_{i}>1$ may still be consistent with RUM (see Börsch-Supan (1990) and Herriges and Kling (1996)).
} 
departure from IIA that allows for the similarity effect (i.e., violations of IAA), our analysis reveals that NSC is the model obtained when IIA is relaxed to allow for the similarity effect.

To see how our axiomatization provides a clearer picture of nested logit, recall its standard textbook description. For instance, Chapter 4 of Train (2009) states that for nested logit "IIA holds over alternatives in each nest and independence of irrelevant nests ${ }^{4}$ (IIN) holds over alternatives in different nests." NSC also satisfies these properties. Indeed, ISA ensures the existence of endogenous nests and imposes exactly these properties on them. But this finding shows that "IIA within a nest + IIN" are not sufficient for a nested logit representation, as ISA is equivalent to NSC. Since there are missing behavioral assumptions behind nested logit, the textbook description is incomplete.

To provide a complete picture of nested logit, we establish two characterizations of nested logit as well as a characterization of random utility nested logit. Our first characterization shows that an NSC is a nested logit if and only if it satisfies Log Ratio Invariance. This axiom requires that the natural logarithm of certain probability ratios featuring collections of similar alternatives is menu-independent. The explicit use of a functional form in the axiom allows us to establish necessary and sufficient conditions for the functional form assumed in nested logit with finite data.

Our second characterization is based upon a novel monotonicity condition, Relative Likelihood Independence, that is necessary for nested logit and becomes sufficient under a mild richness assumption. To understand this axiom, note that nested logit (Equation 2) requires that the attractiveness of a nest is increasing in the sum of utilities. Relative Likelihood Independence implies that this feature must hold in a relative sense; the attractiveness of a nest relative to another nest is increasing in the sum of utilities, holding the alternatives in the other nest fixed. Finally, random utility nested logit is characterized by one additional axiom, Regularity, a wellknown monotonicity property that all random utility models must satisfy (under the same richness assumption). We summarize all of our characterization results in Table 1.

\begin{tabular}{|lll|}
\hline NSC & $\Leftrightarrow$ ISA & \\
\hline Luce & $\Leftrightarrow$ ISA + IAA & $\Leftrightarrow$ IIA \\
\hline Nested Logit & $\Leftrightarrow$ ISA + LRI & $\Leftrightarrow$ ISA + RLI (Richness) \\
\hline RU Nested Logit & $\Leftrightarrow$ & Nested Logit + Regularity (Richness) \\
\hline
\end{tabular}

Table 1: Summary Of Characterization Results.

Independence of Symmetric Alternatives implies that the revealed similarity relation $\sim_{p}$ is transitive, which ensures that the nests form a partition. Hence, our axiomatic characterizations show that the notion of (categorical) similarity in nested logit, as well as in NSC, is quite structured. In some applications, an analyst may want to allow for more flexible forms of substitutability. For this reason, cross-nested logit, a generalization of nested logit, has been proposed and widely applied in empirical work (see Vovsha (1997), Ben-Akiva and Bierlaire (1999)). The main difference

\footnotetext{
${ }^{4}$ If $a$ and $b$ are from distinct nests, then the addition of an alternative $c$ from a third nest will not affect the relative probabilities of $a$ and $b$.
} 
between nested logit and cross-nested logit is that alternatives may belong to several nests in crossnested logit. Since we are focused on the problem of recovering the nesting structure, we consider a generalization of cross-nested logit that relaxes the typical parameter restriction, and refer to this as the unrestricted cross-nested logit. We show that the unrestricted cross-nested logit does not have testable implications. Therefore, our results reveal the trade-off between nested logit and cross-nested models: relaxing the partition structure of nested logit results in an overly permissive model. In other words, the behavioral content of cross-nested logit is essentially driven by the analyst's assumption of the nest structure and parameter restrictions.

In practice, $p$ is estimated from observed choices and "IIA like" conditions never hold exactly. However, we show that the true nest structure can still be identified, for any NSC, by solving a minimization problem. In particular, our axiomatic characterization allows us to derive a "distance" function $D$ that measures, for a given nest structure, the degree of violations of IIA within and across nests for a given set of observations. When the data are close to the true (or theoretical) $p$, the true nest structure will be the unique minimizer of $D$. In applied settings where the researcher has several nest structures in mind, $D$ may also be useful as a selection criteria.

Because the number of possible nests grows exponentially as the number of alternatives increases, the full minimization problem may become intractable quickly. However, this issue can be managed due to insights from our similarity relation; one only needs to check nest structures that are consistent with an empirical approximation of $\sim_{p}$. In fact, we show that there are at most $|X|$ potential nests that we need to check, where $|X|$ is the number of alternatives. We illustrate our theoretical finding and our data-driven algorithm to reduce the number number of candidate nests with a simulation exercise.

The rest of the paper is organized as follows. In section 2, we discuss setup and notation as well as define NSC and nested logit. In section 3, we define revealed similarity and the similarity effect (3.1) before characterizing NSC (3.2) and nested logit (3.3). We discuss ways of extending our notion of similarity and the testable implications of unrestricted cross-nested logit in section 4 . The identification of nest structure from choice data is presented in section 5 . We conclude with a

discussion of related literature in section 6 . We also discuss the relationship between the similarity effect and regularity in Appendix B.1.

\section{Nested Stochastic Choice and Nested Logit}

All of our models are developed in the standard stochastic choice setup. Accordingly, let $X$ be a finite set of alternatives and $\mathscr{A}$ be the collection of all nonempty subsets of $X$ (menus). Let $\mathbb{R}_{+}$ $\left(\mathbb{R}_{++}\right)$denote the non-negative (positive) real numbers.

Definition 1. A function $p: X \times \mathscr{A} \rightarrow[0,1]$ is a stochastic choice function if for any $A \in \mathscr{A}$, $\sum_{a \in A} p(a, A)=1$ and $p(x, A)=0$ when $x \notin A$. In some instances, we may write $p(B, A)=$ $\sum_{b \in B} p(b, A)$ for $B \in \mathscr{A}$. 
Throughout this paper, we assume that $p$ is positive; i.e., $p(a, A)>0$ for all $A \in \mathscr{A}$ and $a \in A$. For notational simplicity, we write $A \cup x$ instead of $A \cup\{x\}$.

The Luce model is the most widely-known and influential stochastic choice model. In this model, choice probabilities are proportional to a utility index: $p(a, A)=u(a) / \sum_{b \in A} u(b)$. In his seminal paper, Luce (1959) proves that a stochastic choice function can be represented by the Luce model if and only if it satisfies IIA for every pair of alternatives.

Definition 2 (IIA). A stochastic choice function $p$ satisfies IIA at $a, b \in X$ if, for any $A \in \mathscr{A}$ with $a, b \in A$,

$$
\frac{p(a, A)}{p(b, A)}=\frac{p(a,\{a, b\})}{p(b,\{a, b\})}
$$

Further, we say that $p$ satisfies IIA if $p$ satisfies IIA at any $a, b \in X$.

It is well known that IIA may fail when similar alternatives are added to the menu, as was illustrated by Debreu's (1960) famous "red bus/blue bus" example. The nested logit is the most commonly applied generalization of Luce's model and was developed to accommodate violations of IIA like the similarity effect. We now formally define nested logit and the NSC, which is a novel, non-parametric version of nested logit.

Definition 3. A stochastic choice function $p$ is a Nested Stochastic Choice (NSC) if there exist a partition $X_{1}, \ldots, X_{K}$ of $X$, a utility function $u: X \rightarrow \mathbb{R}_{++}$, and a nest utility function $v: \bigcup_{i=1}^{K} 2^{X_{i}} \rightarrow \mathbb{R}_{+}$with $v(\emptyset)=0$ such that for any $A \in \mathscr{A}$ and $a \in A \cap X_{i}$,

$$
p(a, A)=\frac{v\left(A \cap X_{i}\right)}{\sum_{j=1}^{K} v\left(A \cap X_{j}\right)} \frac{u(a)}{\sum_{b \in A \cap X_{i}} u(b)} .
$$

Moreover, $p$ is a nested logit if there exist real numbers $\eta_{1}, \ldots, \eta_{n}>0$ such that for any $A \in \mathscr{A}$ and $i \leq K$,

$$
v\left(A \cap X_{i}\right)=\left(\sum_{x \in A \cap X_{i}} u(x)\right)^{\eta_{i}}
$$

Finally, we say that $p$ is a random utility (RU) nested logit when $\eta_{i} \leq 1$ for each $i \leq K$.

The NSC is defined by two Luce procedures, where $v$ governs the choice over nests (e.g., transportation modes or neighborhoods) and $u$ governs the choice over the particular alternatives in the selected nest (e.g., the red/blue bus or a specific apartment). Note that the nest value function $v$ is not necessarily related to alternative utilities $u$, which enables the NSC to capture rich behavior (see 3.4). Despite this generality, the NSC may be falsified with relatively few observations. This is because behavior is disciplined by $u$ and the partition structure of the nests, both of which are menu-independent. ${ }^{5}$

\footnotetext{
${ }^{5}$ It is straightforward to derive from the representation that for any $A \subseteq X$ with $|A|=3$, there is a distinct pair $a, b \in A$ such that $\frac{p(a,\{a, b\})}{p(b,\{a, b\})}=\frac{p(a, A)}{p(b, A)}$. This is because for any three alternatives, either (i) at least two belong to the same nest or (ii) all three belong to distinct nests. Hence there must exist some pair for which IIA holds and therefore NSC may be rejected with only three alternatives.
} 
The nested logit imposes a specific parametric relationship between $v$ and $u$. Notice that the NSC, and consequently nested logit, reduces to the Luce model when there is a single nest. Additionally, it is simple to see from Definition 3 that any NSC satisfies "IIA within a nest + IIN." These properties are often taken as the hallmark of nested logit, yet they apply to all NSC (with endogenous nests). Since NSC permits behavior that nested logit does not (three examples are discussed in section 3.4), this means that there are additional behavioral assumptions underpinning nested logit. We elucidate these assumptions in section 3.3 .

In nested logit, $1-\eta_{i}$ is usually considered a measure of correlation or substitutability between alternatives in nest $i$. When $\eta_{i}<1$ alternatives within the same nest are substitutes. Further, it is well known that when $\eta_{i}<1$, the nested logit is always a RUM for any profile of utilities.

When $\eta_{i}>1$, choice frequencies may (but do not always) violate regularity, a necessary property of every random utility model (RUM) which states that the probability of choosing some alternative must never increase as the menu expands. ${ }^{6}$ Behaviorally, we can interpret $\eta_{i}>1$ as indicating complementarities among alternatives. ${ }^{7}$ In certain contexts, we may even anticipate $\eta_{i}>1$. For instance, Foubert and Gijsbrechts (2007) study the effects of "product bundling" and find a parameter greater than one, consistent with the effectiveness of bundling. ${ }^{8}$ As NSC allows for violations of regularity, formally defined below, the NSC is not nested by RUM.

Definition 4 (Regularity). A stochastic choice function $p$ satisfies regularity if $p(x, A \cup y) \leq$ $p(x, A)$ for any $A \in \mathscr{A}$ and $x, y \in X$ with $x \in A$.

Finally, note that when $\eta_{i}=1$ the nest value is exactly proportional to the sum of Luce utilities. If this proportionality happens for every nest $i$, the model reduces to Luce. In fact, in this case the Luce model has multiple NSC (and nested logit) representations with different partitions and identification of a unique nest structure is not possible. To rule this out, we say that an NSC $p$ with $\left(v, u,\left\{X_{i}\right\}_{i=1}^{K}\right)$ is nondegenerate if there is at most one nest where this proportionality occurs: there is at most one $i \leq K$ such that for some $a \in X_{i}$,

$$
\frac{\sum_{x \in A_{i}} u(x)}{v\left(A_{i}\right)}=\frac{u(a)}{v(a)} \text { for any } A_{i} \subseteq X_{i} \text { with } a \in A_{i} .
$$

This restriction rules out cases when $v$ is always proportional to the sum of Luce utilities. Further, the Luce model has a unique nondegenerate NSC representation in which there is a single nest, $X_{1}=X{ }^{9}$ This nondegeneracy condition will be crucial for the unique identification of nests, but

\footnotetext{
${ }^{6}$ There is some experimental evidence that violations of regularity occur when similar alternatives are introduced, in-line with convex aggregation. This has been observed in humans (Rieskamp, Busemeyer, and Mellers, 2006) and animals (Shafir, Waite, and Smith, 2002). Recently, Batley and Hess (2016) estimated nested logit parameters to check for consistency with regularity (and various forms of stochastic transitivity) and found that parameter values consistent with violations of regularity provided the best fit.

${ }^{7}$ Relatedly, Cerreia-Vioglio, Dillenberger, Ortoleva, and Riella (2019) suggests that regularity may be violated due to deliberate randomization between complementary lotteries.

${ }^{8}$ Indeed, in regard to whether $\eta_{i}$ should be less than or greater than one, Train, McFadden, and Ben-Akiva (1987) state that "...the value of $\left[\eta_{i}\right]$ indicates relative substitutability within and among nests, and neither possibility can be rule out a priori."

${ }^{9}$ Indeed, if there are $i, j$ such that $\frac{\sum_{x \in A_{i}} u(x)}{v\left(A_{i}\right)}=\frac{u(a)}{v(a)}$ and $\frac{\sum_{y \in A_{j}} u(y)}{v\left(A_{j}\right)}=\frac{u(b)}{v(b)}$ for any $A_{i} \subseteq X_{i}, A_{j} \subseteq X_{j}, a \in A_{i}$,
} 
it is not required for the sufficiency part of our characterization (Theorem 1).

\section{Behavioral Characterizations}

\subsection{The Similarity Effect and Revealed Similarity}

Following the intuition behind the similarity effect, we introduce a notion of revealed similarity that will be essential to our analysis. Consider the effect of adding an alternative $x$ on the probabilities of choosing $a$ and $b$ from some menu $A$. Adding $x$ might decrease these probabilities as it competes with $a$ and $b$. If $x$ disproportionately affects one of them, say $a$ relative to $b$, this reveals that $a$ and $b$ are dissimilar. Conversely, if $x$ takes away from $a$ and $b$ proportionally, then this reveals that $a$ and $b$ are similar (symmetric) in menu $A$. We take a conservative approach and call two alternatives similar only if this is true for any menu $A$ (they are symmetric to all other alternatives).

Definition 5. For any alternatives $a, b \in X$, we say that $a$ and $b$ are revealed categorically similar, denoted by $a \sim_{p} b$, if $p$ satisfies IIA at $a, b$. We also say that $a$ and $b$ are revealed categorically dissimilar if $a \nsim_{p} b$.

The similarity effect is often defined using an exogenously given similarity relation. With our formal notion of revealed similarity, we may establish a fully behavioral definition of the similarity effect given $\sim_{p} \cdot{ }^{10}$ Recalling the red bus/blue bus example, adding the blue bus had a larger effect on the red bus than on the train. Hence the blue bus "takes more away" from similar alternatives than from dissimilar alternatives.

Definition 6. A stochastic choice function $p$ exhibits the similarity effect if for all $A \in \mathscr{A}$, $a, b \in A$, and $x \notin A$,

$$
\text { if } a \sim_{p} x \text { and } b \varkappa_{p} x \text {, then } \frac{p(a, A \cup x)}{p(b, A \cup x)}<\frac{p(a, A)}{p(b, A)}
$$

Intuitively, $x$ hurts the revealed categorically similar alternative $a$ more than a revealed categorically dissimilar alternative $b$. Since $a$ and $x$ are closer substitutes, $x$ competes more with $a$ than it does with $b$.

\subsection{Characterization of NSC}

In order to introduce our axiom, we consider the general effect of introducing an alternative $x$ on the choice probabilities of two alternatives $a$ and $b$. IIA requires that the relative probability between $a$

and $b \in A_{j}$, then $X_{i} \cup X_{j}$ should be treated as one nest. It is also not difficult to show that the set of degenerate NSC is measure zero with respect to the set of all NSC.

${ }^{10}$ There are other ways to define similarity and other properties one might demand of a similarity relation. For instance, Rubinstein (1988) studies similarity and choice under risk. In his paper, the similarity relation is reflexive and symmetric, like ours, but also must satisfy a form of betweenness with respect to objective attributes and violates transitivity, unlike ours. Natenzon (2018) introduces a notion of comparative similarity based on absolute rather than relative choice frequencies. This similarity notion is not related to IIA and will not induce a partition structure on the set of alternatives. 
and $b$ is always independent of $x$. However, as the similarity effect suggests, (asymmetric) similarity between $x$ and $a, b$ might affect the relative probabilities. We therefore divide IIA into two logically independent axioms based on the revealed similarity between $x$ and $a, b$.

Axiom 1 (Independence of Symmetric Alternatives). For any $A \in \mathscr{A}, a, b \in A$, and $x \notin A$,

$$
\begin{aligned}
& a \sim_{p} x \text { and } b \sim_{p} x \\
& \quad \text { or } \\
& a \nsim_{p} x \text { and } b \varkappa_{p} x
\end{aligned} \quad \Longrightarrow \quad \frac{p(a, A)}{p(b, A)}=\frac{p(a, A \cup x)}{p(b, A \cup x)} .
$$

Axiom 2 (Independence of Asymmetric Alternatives). For any $A \in \mathscr{A}, a, b \in A$, and $x \notin A$,

$$
a \sim_{p} x \text { and } b \varkappa_{p} x \quad \Longrightarrow \quad \frac{p(a, A)}{p(b, A)}=\frac{p(a, A \cup x)}{p(b, A \cup x)}
$$

The first axiom requires that the relative probability between $a$ and $b$ is independent of $x$ when $a$ and $b$ are revealed categorically (dis)similar to $x$. Intuitively, if $a$ and $b$ are symmetric from the perspective of $x$, then $x$ should symmetrically influence $a$ and $b$; it does not affect the relative probability between $a$ and $b$. The similarity effect directly contradicts the second axiom yet is unrelated to the first axiom.

Observation 1. Luce's IIA is equivalent to the joint assumption of Independence of Symmetric Alternatives and Independence of Asymmetric Alternatives. Moreover, the two axioms are independent.

We show in Theorem 1 that NSC is characterized by Independence of Symmetric Alternatives, and thus NSC is precisely the generalization of Luce's model that accommodates the similarity effect.

Theorem 1. Let $p$ be a stochastic choice function with at least three alternatives that are dissimilar to each other. Then $p$ satisfies Independence of Symmetric Alternatives if and only if $p$ is a nondegenerate NSC.

Theorem 1 characterizes NSC when there are at least three nests; $a \varkappa_{p} b, b \varkappa_{p} c$, and $a \varkappa_{p} c$ for distinct alternatives. ${ }^{11}$ While the proof is in the appendix, we discuss briefly how our axiom characterizes NSC. It should be apparent from the definition that $\sim_{p}$ is reflexive and symmetric. It turns out that the first part of Independence of Symmetric Alternatives $\left(a \sim_{p} x\right.$ and $\left.b \sim_{p} x\right)$ implies that $\sim_{p}$ is transitive. ${ }^{12}$ Hence, transitivity of $\sim_{p}$ immediately generates a partition $X_{1}, \ldots, X_{k}$ of $X$

\footnotetext{
${ }^{11}$ When the assumption is violated (i.e., there are only two nests), we can still obtain the characterization result by modifying Independence of Symmetric Alternatives. In particular, we can impose a modification of Luce's (1959) Product Rule instead of the second part of Independence of Symmetric Alternatives. It is well known that IIA is equivalent to the Product Rule for menus with two alternatives (see Luce (1959)).

${ }^{12}$ More general notions of similarity may be intransitive (e.g., due to context dependence). Since we take a conservative definition of similarity, we find transitivity quite reasonable in our setting. That is, by requiring $\frac{p(a, A)}{p(b, A)}=$ $\frac{p(a,\{a, b\})}{p(b,\{a, b\})}$ for any menu, we eliminate much of the context dependence. Further, transitivity of this revealed similarity relation is implicitly assumed in nested logit. See section 4.
} 
(or disjoint nests) such that any two alternatives in $X_{i}$ are revealed categorically similar. ${ }^{13}$ However, by itself it imposes no particular structure on choice, nor does it establish a relationship between the partition and choices (except that IIA is satisfied within each nest). The essential structure of NSC is captured by the second part of Independence of Symmetric Alternatives $\left(a \nsim_{p} x\right.$ and $\left.b \nsim_{p} x\right)$. Therefore, almost all of the proof is devoted to showing that the second part of Independence of Symmetric Alternatives implies a nested choice structure consistent with this partition.

Lastly, we state the uniqueness properties of the NSC representation. The following proposition shows that the nest structure is unique, the nest utility $v$ is unique up to a positive scalar, and Luce's utility $u$ is unique up to a positive scalar at each nest.

Proposition 1 (Uniqueness). Suppose $p$ is a nondegenerate $N S C$ with respect to $\left(v, u,\left\{X_{i}\right\}_{i=1}^{K}\right)$ as well as to $\left(v^{\prime}, u^{\prime},\left\{X_{i}^{\prime}\right\}_{i=1}^{K^{\prime}}\right)$. Then $K=K^{\prime}$ and $\left\{X_{i}\right\}_{i=1}^{K}=\left\{X_{i}^{\prime}\right\}_{i=1}^{K^{\prime}}$. Moreover, there is $\left(\alpha_{1}, \ldots, \alpha_{K}, \delta\right) \in \mathbb{R}_{++}^{K+1}$ such that $v^{\prime}=\delta v$ and for any $x_{i} \in X_{i}, u^{\prime}\left(x_{i}\right)=\alpha_{i} u\left(x_{i}\right)$.

\subsection{Characterizations of Nested Logit}

The most well-known special case of NSC is nested logit, which was specifically created to handle the similarity effect. The difference between NSC and nested logit is that the latter imposes a special structure on the nest values, $v\left(A \cap X_{i}\right)=\left(\sum_{a \in A \cap X_{i}} u(a)\right)^{\eta_{i}}$ with $\eta_{i}>0$, which has non-trivial behavioral consequences.

Despite its widespread use, nested logit has not been subject to careful axiomatic analysis in the way that other choice models have been. We provide two characterizations of nested logit that clarify the behavioral assumptions embedded in this model. The first characterization uses one additional axiom that imposes a menu independence condition on certain probability ratios.

Axiom 3 (Log Ratio Invariance). For any $a, x \in X$ and $A, B \in \mathscr{A}$ such that $a \sim_{p} a^{\prime}$ for all $a^{\prime} \in A \cup B$,

$$
\frac{\log \left(\frac{p(A, A \cup x)}{p(x, A \cup x)} / \frac{p(a,\{a, x\})}{p(x,\{a, x\})}\right)}{\log \left(\frac{p(A, A \cup a)}{p(a, A \cup a)}\right)}=\frac{\log \left(\frac{p(B, B \cup x)}{p(x, B \cup x)} / \frac{p(a,\{a, x\})}{p(x,\{a, x\})}\right)}{\log \left(\frac{p(B, B \cup a)}{p(a, B \cup a)}\right)} .
$$

Log Ratio Invariance requires that the ratio $\log \left(\frac{p(A, A \cup x)}{p(x, A \cup x)} / \frac{p(a,\{a, x\})}{p(x,\{a, x\})}\right)$ and $\log \left(\frac{p(A, A \cup a)}{p(a, A \cup a)}\right)$ are proportional.

Theorem 2. A nondegenerate NSC satisfies Log Ratio Invariance if and only if it is an nested logit.

The explicit use of a functional form in Log Ratio Invariance allows us to establish testable implications for the functional form assumed in nested logit even with finite data.

In the rest of this section, we discuss an alternative axiom that captures the essential features of nested logit without an explicit functional form and shows that is characterizes nested logit under a

\footnotetext{
${ }^{13}$ Transitivity of $\sim_{p}$ is imposed in $\mathrm{Li}$ and Tang (2016), which will be carefully discussed in section 6 . A weak version of transitivity of $\sim_{p}$ is also used in Echenique et al. (2018).
} 
richer domain assumption. To state this axiom, first notice that an important behavioral property of nested logit, beyond its treatment of similarity (ISA), is that the probability of choosing a nest depends on the total attractiveness of the nest: $v\left(A \cap X_{i}\right)$ is increasing in $\sum_{a \in A \cap X_{i}} u(a)$.

This behavior is characterized by a simple monotonicity property imposed among similar alternatives. Suppose that $A, A^{\prime} \in \mathscr{A}$ are menus such that all alternatives in $A \cup A^{\prime}$ are revealed similar. When $A$ is more attractive than $A^{\prime}$, then alternatives in $A$ are always chosen more frequently than alternatives in $A^{\prime}$ when they are compared with any other alternative $x$. More formally, $p\left(A, A \cup A^{\prime}\right) \geq p\left(A^{\prime}, A \cup A^{\prime}\right)$ implies $p(A, A \cup x) \geq p\left(A^{\prime}, A^{\prime} \cup x\right)$ for any $x \notin A \cup A^{\prime}$. This can be viewed as an additional form of context independence, as it requires that there is no interaction between alternatives in $A \cup A^{\prime}$ and $x$ which might create a choice frequency reversal.

Because nested logit involves a power function, it satisfies a stronger version of the monotonicity property above. In particular, the monotonicity property holds even in relative terms: if $A$ is relatively more attractive than $A^{\prime}$ when they are compared to any other menus, $B$ and $B^{\prime}$, then alternatives in $A$ will be chosen relatively more frequently than $A^{\prime}$ when they are chosen against $x$.

Axıom 4 (Relative Likelihood Independence). For any $x \in X$ and $A, B, A^{\prime}, B^{\prime} \in \mathscr{A}$ such that $a \sim_{p} a^{\prime}$ for any $a, a^{\prime} \in A \cup B \cup A^{\prime} \cup B^{\prime}$,

$$
\frac{p(A, A \cup B)}{p(B, A \cup B)} \geq \frac{p\left(A^{\prime}, A^{\prime} \cup B^{\prime}\right)}{p\left(B^{\prime}, A^{\prime} \cup B^{\prime}\right)} \Longrightarrow \frac{p(A, A \cup x)}{p(x, A \cup x)} / \frac{p(B, B \cup x)}{p(x, B \cup x)} \geq \frac{p\left(A^{\prime}, A^{\prime} \cup x\right)}{p\left(x, A^{\prime} \cup x\right)} / \frac{p\left(B^{\prime}, B^{\prime} \cup x\right)}{p\left(x, B^{\prime} \cup x\right)}
$$

In our next result, we prove that Relative Likelihood Independence is a necessary condition for nested logit. Moreover, it implies that $v\left(A \cap X_{i}\right)$ is increasing in $\sum_{a \in A \cap X_{i}} u(a)$.

Proposition 2. Any nested logit satisfies Relative Likelihood Independence. Conversely, if a nondegenerate NSC with $\left(v, u,\left\{X_{i}\right\}_{i=1}^{K}\right)$ satisfies Relative Likelihood Independence, then for each $i \leq K$ there is an increasing function $f_{i}: \mathbb{R}_{++} \rightarrow \mathbb{R}_{++}$such that $v(A)=f_{i}\left(\sum_{x \in A} u(x)\right)$ for any $A \subseteq X_{i}$.

While Relative Likelihood Independence is not sufficient for nested logit, this is essentially due to the limitations of finite data. Indeed, we show that Relative Likelihood Independence characterizes nested logit when the following richness condition is satisfied.

Axiom 5 (Richness). For any $a \in X$ and $\rho \in(0,1)$, there is $b \in X$ such that $a \sim_{p} b$ and $p(a,\{a, b\})=\rho$.

On its own, Richness is relatively mild and simply ensures that there are alternatives for each utility value. However, under this condition Relative Likelihood Independence yields the wellknown functional form of nested logit. Consequently, Relative Likelihood Independence captures all remaining behavioral features of nested logit.

Theorem 3. Any nondegenerate NSC that satisfies Relative Likelihood Independence and Richness is a nested logit. 
In applied settings, nested logit is often restricted to $\eta_{i} \in(0,1]$, as this is sufficient for it to be a RUM. Since any RUM satisfies Regularity, a random utility nested logit must as well. It is well known that Regularity is necessary but not sufficient for a model to be a RUM in general. However, we show that Regularity is sufficient for the nested logit to be a RUM under Richness.

Proposition 3. Any nested logit that satisfies Regularity and Richness is a random utility nested logit.

If $\eta_{i}>1$, nested logit will violate regularity for some specifications of $u$. Therefore Richness strengthens the bite of Regularity and $\eta_{i} \in(0,1]$ is ensured.

\subsection{Beyond Nested Logit}

It is a matter of folk knowledge that the aforementioned "IIA within a nest and IIN" properties serve as the behavioral underpinnings of nested logit. However, our results show that "IIA within a nest and IIN" (with an endogenous nest structure) in fact characterize NSC, and nested logit requires an additional property (Relative Likelihood Independence). In this subsection, we present three special cases of NSC that are distinct from nested logit. These examples illustrate some natural choice behaviors that Relative Likelihood Independence rules out, further clarifying the behavioral assumptions behind nested logit.

\subsubsection{Linear NSC}

One interesting example of NSC that is distinct from nested logit is the Linear NSC. In this example, the nest value is linear in total nest utility, in contrast to the power function used in nested logit. For each nest $i$, there exist parameters $\lambda_{i} \geq 0$ and $\nu_{i}$, so that

$$
v\left(A \cap X_{i}\right)=\lambda_{i}\left(\sum_{x \in A \cap X_{i}} u(x)\right)+\nu_{i}
$$

In the Linear NSC, the attractiveness of a nest depends on both its instrumental utility, through $\lambda_{i}$, and an intrinsic "category" utility, through $\nu_{i}$. It turns out that the Linear NSC is a special case of both Elimination-by-Aspects (EBA) of Tversky (1972) and the Attribute Rule (AR) of Gul, Natenzon, and Pesendorfer (2014). Consequently, the Linear NSC is also a RUM.

\subsubsection{Menu-Dependent Substitutability}

In nested logit, the nest parameter $\eta_{i}$ captures substitutability of alternatives. While the standard nested logit only allows for a single substitution parameter for each nest, NSC can accommodate menu-dependent substitutability. For instance, consider the following example where substitutability depends on the size of the menu, capturing the idea that consumers may find it more difficult to distinguish between alternatives in larger option sets. 
For each nest $i$, there exists a threshold $\tau_{i} \in\left\{1, \ldots,\left|X_{i}\right|\right\}$, and nest parameters $\eta_{i}, \tilde{\eta}_{i}>0$, so that

$$
v\left(A \cap X_{i}\right)= \begin{cases}\left(\sum_{x \in A \cap X_{i}} u(x)\right)^{\eta_{i}} & \text { if }\left|A \cap X_{i}\right|>\tau_{i} \\ \left(\sum_{x \in A \cap X_{i}} u(x)\right)^{\tilde{\eta}_{i}} & \text { if }\left|A \cap X_{i}\right| \leq \tau_{i}\end{cases}
$$

If $1-\eta_{i}>1-\tilde{\eta}_{i}$, this means the agent perceives fewer differences among alternatives as the nest becomes "more represented." That is, when $\left|A \cap X_{i}\right|$ exceeds $\tau_{i}$, alternatives are "more substitutable." In this example, $\tau_{i}$ has a natural interpretation as the consumer's "distinction capacity." Further, if $1-\eta_{i}>0>1-\tilde{\eta}_{i}$, then whether the alternatives are complements or substitutes depends on the size of the nest. Lastly, when $\eta_{i}$ and $\tilde{\eta}_{i}$ are both less than one and are sufficiently close, this example is also consistent with RUM.

\subsubsection{Attention and Spillover Effects}

The NSC also allows for cases where the nest value is not directly tied to alternative utility. We consider a particular example in which $v$ is determined by the "salience" of alternatives. For some function $S: X \rightarrow \mathbb{R}_{++}$,

$$
v\left(A \cap X_{i}\right)=\max _{x \in A \cap X_{i}} S(x)
$$

In this specification, the value of a nest is determined by the "attractiveness" or "noticeability" of its most salient alternative. To illustrate its behavioral implications, suppose there are three alternatives, $X=\{x, y, z\}$, with nests $X_{1}=\{x, z\}$ and $X_{2}=\{y\}$. When $z$ is highly salient but low utility, $S(z) u(x)>S(x)[u(x)+u(z)]$, then $\frac{p(x,\{x, y, z\})}{p(y,\{x, y, z\})}>\frac{p(x,\{x, y\})}{p(y,\{x, y\})}$. Examples of such $z$ include brands offering a high-end good with a high price to attract attention, expecting all consumers to purchase their "moderate" offering $x$. When the value of $S(z)$ is large enough relative to the value of $u(z)$, this may induce a violation of regularity. Similar examples can generate "spillover" effects. For example, one successful or attractive product may funnel attention to others, causing demand spillover. This is the traditional rationale behind the use of "loss-leaders" (Lal and Matutes (1994)) or "attention-grabbers" (Eliaz and Spiegler (2011)).

\section{Revealed Similarity and its Extensions}

We say that two alternatives are revealed categorically similar if IIA is satisfied between them at all menus. Requiring this eliminates the menu dependence of similarity, and so our notion of revealed similarity captures a form of absolute or fundamental similarity. Consequently, similarity is symmetric and, under Independence of Symmetric Alternatives, transitive. One drawback is that this notion does not allow statements about comparative similarity; two alternatives are similar or 
not. Additionally, in some cases impressions of similarity may be context-dependent. ${ }^{14}$ Because of these apparent limitations, we consider two ways in which to extend NSC to accommodate more complex notions of similarity.

The first extension of NSC relaxes the requirement that an alternative must belong to a single nest. In section 4.1, we consider the (unrestricted) cross-nested logit (Vovsha, 1997) and the (more general) generalized nested logit (Wen and Koppelman (2001)), which allow for each alternative to be "allocated" across several nests. While overlapping nests allows for the most flexible notion of similarity, these models have no testable implications if the nests are not known a priori and parameter values are unrestricted. Thus we demonstrate an important trade-off between nested and cross-nested models.

The second extension of NSC allows for "intermediate nests." These intermediate nests are often visually represented through a multi-level decision tree. Within this structure, we can allow for a more nuanced notion of similarity through the introduction of a second similarity relation that is conceptually related to our core similarity notion. This secondary relation captures "context-dependent" similarity and allows for comparative statements. We provide an axiomatic characterization of this model (Theorem 6) in appendix B.3. ${ }^{15}$

\subsection{Overlapping Nests}

In NSC, each alternative belongs to one, and only one, nest. This feature of NSC places restrictions on the similarity relation. Because of these restrictions, in some settings, applied researchers have proposed allowing alternatives to exist in multiple nests. This leads to a class of models known as "cross-nested logits" (see Vovsha (1997), Ben-Akiva and Bierlaire (1999), Wen and Koppelman (2001), Papola (2004), and Bierlaire (2006)). In the cross-nested logit and the generalized nested logit, each alternative is allocated among the various nests. This allocation is specified with a vector of weights, one for each alternative, which describes to what extent an alternative belongs to each nest.

We show that any stochastic choice rule $p$ can be rationalized by some unrestricted crossnested logit. That is, for any $p$, there exist some nesting structure, $X_{1}, \ldots, X_{K}$, allocations to these nests, $\left(\alpha_{x}^{k}\right)_{k=1}^{K}$, and utilities so that the resulting unrestricted cross-nested logit generates identical choice frequencies. Hence, unlike the nested logit and Luce models, there can be no behavioral characterization of the unrestricted cross-nested logit. The only testable implications of the model are due to the analysts' assumptions about alternative categorization and parameter restrictions.

\footnotetext{
${ }^{14}$ There is a sense in which our notion is somewhat moderate. Consider Debreu's red bus/blue bus example. In this case, the similar alternatives (buses) are in fact identical, often called duplicates (or in some cases replicas). Duplicates are not merely similar alternatives; they are similar and provide the exact same utility value. For example, in Gul et al. (2014) the use of duplicates is essential to their characterization of the Attribute Rule. Formally, $x$ and $y$ are duplicates if $p(a, A \cup x)=p(a, A \cup y)$ for any $A$ and $a \in A$. However, our notion of similarity is not tied to utility. A commuter may regard all buses as similar (i.e., they belong to the same nest), yet nothing in our model restricts an agent from exhibiting a preference over different buses (e.g., because some bus routes may be faster or cheaper than others).

${ }^{15}$ Just as our similarity relation identifies endogenous nests, this secondary relation identifies endogenous, intermediate nests. Thus, Theorem 6 shows that we may identify an endogenous tree structure.
} 
Definition 7 (Generalized Nested Logit). A stochastic choice function $p$ is an unrestricted generalized nested logit if there is a collection of subsets $X_{1}, \ldots, X_{K}$ of $X$ and a vector $\left(\alpha_{x}^{k}\right)_{k=1}^{K} \in \mathbb{R}_{+}^{K}$ with $\sum_{k=1}^{K} \alpha_{x}^{k}=1$ for each $x \in X$ such that $x \notin X_{k}$ iff $\alpha_{x}^{k}=0$, a utility function $u: X \rightarrow \mathbb{R}_{++}$, and parameters $\left(\lambda_{k}\right)_{k=1}^{K} \in \mathbb{R}_{++}^{K}$ such that for any $A \in \mathscr{A}$ and $x \in A$,

$$
p(x, A)=\sum_{k: x \in A \cap X_{k}} \frac{\left(\alpha_{x}^{k} u(x)\right)^{\frac{1}{\lambda_{k}}}}{\sum_{y \in A \cap X_{k}}\left(\alpha_{y}^{k} u(y)\right)^{\frac{1}{\lambda_{k}}}} \cdot \frac{\left(\sum_{y \in A \cap X_{k}}\left(\alpha_{y}^{k} u(y)\right)^{\frac{1}{\lambda_{k}}}\right)^{\lambda_{k}}}{\sum_{l: A \cap X_{l} \neq \emptyset}\left(\sum_{z \in A \cap X_{l}}\left(\alpha_{z}^{l} u(z)\right)^{\frac{1}{\lambda_{l}}}\right)^{\lambda_{l}}} .
$$

Moreover, we say that $p$ is an unrestricted cross-nested logit if $\lambda_{k}=\lambda_{k^{\prime}}$ for any $k, k^{\prime} \leq K$.

Theorem 4. Every stochastic choice function is an unrestricted cross-nested logit.

Corollary 1. Every stochastic choice function is an unrestricted generalized nested logit.

Our result relies on the key insight that the cross-nested logit is behaviorally equivalent to a form of menu-dependent utility. We first prove this equivalence as Lemma 1 and show how we can go from menu-dependent utility to weighted allocations and back. This equivalence between the cross-nested logit and menu-dependent utility allows us to reduce the problem of finding weights to the problem of finding menu-dependent utility values for each menu that satisfy the cross-nested logit equation. The bulk of the proof is dedicated to showing that the existence of these menudependent utilities is equivalent to the existence of a fixed point for some self-map. The proof is completed by applying Brouwer's fixed point theorem.

This result precisely shows the trade-offs between using nested logit and related models: either accept a restrictive form of similarity or impose assumptions on nest structure and model parameters. As we mentioned previously, further assumptions on parameters or nest structure may lead to testable restrictions. In the literature, similar to nested logit, it is commonly assumed that $\lambda \leq 1$, since this is sufficient for cross-nested logit to be RUM. As with our handling of nested logit, we refer to such specifications as the random utility cross-nested logit. Note that our result shows that this restriction is not necessary for consistency with RUM; By Theorem 4, every RUM has an unrestricted cross-nested logit representation with $\lambda>1$.

In any case, a random utility cross-nested logit must have, at least, the same testable restrictions as RUM. However, our result suggests that random utility cross-nested logit may not have any testable restrictions beyond RUM. In fact, although it does not prove our hypothesis, Fosgerau, McFadden, and Bierlaire (2013) prove that the set of random utility cross-nested logit models is dense in the set of RUMs. Note that our Theorem 4 is quite different from the result of Fosgerau et al. (2013) for the following reasons: (i) we prove an exact result while they prove an approximation result, (ii) they focus on random utility cross-nested logit, and (iii) our proof techniques are completely different because their proof relies on the properties of the CDF for GEV distributions while we use Brouwer's fixed point theorem. 


\section{$5 \quad$ Identifying Nests}

In most applications of nested logit to market data, researchers assume nests based on knowledge of alternative attributes. This is potentially problematic, as in many environments there are many plausible structures. When studying vehicle choice, the researcher might construct nests based on brand, body type (e.g., sedan vs. truck), or country of origin. ${ }^{16}$ In other environments, classification may be subjective. When studying choice over apartments, nests might depend on both observable attributes and a myriad of unobservables: subjective impressions of neighborhoods, proximity to landmarks, or a host of other features. If the nest structure is misspecified, this may lead to biased conclusions regarding substitutability of goods and systematically inaccurate predictions. ${ }^{17}$

We show in subsection 5.1 that the true (unobserved) nest structure can be identified from the data by solving a minimization problem. Any potential nest structure has implications for when IIA may and may not be violated between alternatives. For a hypothesized nest structure, $\mathcal{Y}$, we propose a measure of the total magnitude of IIA violations within and across the proposed nests, $D(\mathcal{Y})$. We show that the true nest is a minimizer of $D$ and that it will be the unique minimizer of $D$ under a mild identification assumption (Proposition 4). In cases where the researcher has several potential nest structures in mind, such as in vehicle choice, our procedure for nest identification could be useful for nest selection. The researcher can calculate $D$ for the particular nests in mind and select the one that best fits.

Because the number of possible nests grows exponentially as the number of alternatives increases, the full minimization problem becomes intractable. However, this issue can be managed due to insights from our similarity relation; by Proposition 5, one only needs to check nest structures that are consistent with an empirical approximation of $\sim_{p}$. Note that in finite data sets it is unlikely that IIA will hold between any alternatives (e.g., since we observe a finite sample from the true distribution). However, one can measure the magnitude of the the IIA violation between $a, b$ across various menus in the data. If this magnitude is below some threshold $\epsilon$, then we conclude that $a$ and $b$ are approximately similar: $a \sim_{\epsilon} b$. When $\sim_{\epsilon}$ is transitive, then there are at most $|X|$ potential nests that we need to check, as stated in Proposition 6.

\subsection{Identifying Nests by Distance Minimization}

To analyze the problem of nest identification, we consider a data set denoted $\mathcal{O}=\left\{A,\left\{p_{t}(\cdot, A)\right\}_{t=1}^{N_{A}}\right\}_{A \in \mathscr{A}}$, where $N_{A}$ is the number of observations of menu $A$ and $p_{t}(a, A)=1$ means that $a$ was chosen from $A$ at observation $t \leq N_{A}$. We also require $\sum_{x \in A} p_{t}(x, A)=1$ for each $A$, so that $p_{t}(a, A)=1$

\footnotetext{
${ }^{16} \mathrm{~A}$ common approach to this type of problem is to utilize multiple levels of nesting (which we characterize in appendix B.3). Even under this approach, the order of the levels matters.

${ }^{17}$ Greene (2003) provides an excellent summary of this issue: "To specify the nested logit model, it is necessary to partition the choice set into branches [nests]. Sometimes there will be a natural partition ... In other instances, however, the partitioning of the choice set is ad hoc and leads to the troubling possibility that the results might be dependent on the branches so defined. ... There is no well-defined testing procedure for discriminating among tree structures, which is a problematic aspect of the model."
} 
implies $p_{t}(b, A)=0$ for any $b \in A \backslash\{a\}$. Note that we may always write

$$
p_{t}(a, A)=\bar{p}(a, A)+\epsilon_{t, a, A},
$$

where $\bar{p}(a, A)$ is the probability that $a$ is chosen from $A$ according to the NSC with $\left(v, u,\left\{X_{i}\right\}_{i=1}^{K}\right)$. Then, the observed choice frequency of $a$ from $A$ in $\mathcal{O}$ is

$$
p(a, A) \equiv \frac{\sum_{t=1}^{N_{A}} p_{t}(a, A)}{N_{A}}=\bar{p}(a, A)+\epsilon_{a, A} \text { where } \epsilon_{a, A} \equiv \frac{\sum_{t=1}^{N_{A}} \epsilon_{t, a, A}}{N_{A}}
$$

We assume that $\left\{p_{t}(\cdot, A)\right\}_{t=1}^{N_{A}}$ are independently drawn according to $\bar{p}(\cdot, A)$. Then, by the classical Glivenko-Cantelli theorem, $\epsilon_{a, A} \stackrel{a . s .}{\longrightarrow} 0 .{ }^{18}$ For notational simplicity, we write

$$
r_{A}\left(A^{\prime}, B^{\prime}\right) \equiv \frac{p\left(A^{\prime}, A\right)}{p\left(B^{\prime}, A\right)} \text { and } \bar{r}_{A}\left(A^{\prime}, B^{\prime}\right) \equiv \frac{\bar{p}\left(A^{\prime}, A\right)}{\bar{p}\left(B^{\prime}, A\right)} \text { for any } A, A^{\prime}, B^{\prime} \in \mathscr{A}
$$

Finally, let $\mathscr{X}$ denote the set of all partitions of $X$ and $\mathcal{X}^{*}$ denote the true partition $\left\{X_{i}\right\}_{i=1}^{K}$.

Consider the following minimization problem.

$(\mathbf{N M P})$

$$
\min _{\mathcal{Y} \in \mathscr{X}} D(\mathcal{Y})=D_{1}(\mathcal{Y})+D_{2}(\mathcal{Y})
$$

$$
D_{1}(\mathcal{Y})=\frac{\sum_{Y \in \mathcal{Y}} \sum_{A, B \in \mathscr{A}, a, b \in A \cap B \cap Y}\left(\log \left(r_{A}(a, b)\right)-\log \left(r_{B}(a, b)\right)\right)^{2}}{\sum_{Y \in \mathcal{Y}}|\{(A, B, a, b) \mid a, b \in A \cap B \cap Y\}|},
$$

$$
D_{2}(\mathcal{Y})=\frac{\sum_{Y, Y^{\prime} \in \mathcal{Y}} \sum_{A, B \in \mathscr{A}: A \cap Y=B \cap Y, A \cap Y^{\prime}=B \cap Y^{\prime}}\left(\log \left(r_{A}\left(Y, Y^{\prime}\right)\right)-\log \left(r_{B}\left(Y, Y^{\prime}\right)\right)\right)^{2}}{\sum_{Y, Y^{\prime} \in \mathcal{Y}}\left|\left\{(A, B) \mid A \cap Y=B \cap Y, A \cap Y^{\prime}=B \cap Y^{\prime}\right\}\right|}
$$

Intuitively, $D_{1}(\mathcal{Y})$ measures the degree to which the data violates IIA among elements in the same nest in $\mathcal{Y}$, while $D_{2}(\mathcal{Y})$ measures the degree to which the data violates IIA among different nests in $\mathcal{Y}$. These measures are motivated by our axiom Independence of Symmetric Alternatives: $D_{1}$ measures the extent to which the first part of Independence of Symmetric Alternatives holds, and $D_{2}$ measures the extent to which the second part of Independence of Symmetric Alternatives holds.

Similarly, let us define loss functions $D^{*}, D_{1}^{*}, D_{2}^{*}$ when there is no observational noise; these are defined by replacing $p$ with $\bar{p}$ in Equations 9-10. Moreover, let $\hat{\mathcal{X}}=\arg \min _{\mathcal{Y} \in \mathscr{X}} D(\mathcal{Y})$. Note that $\hat{\mathcal{X}}$ is an M-estimator (Amemiya (1985)). Hence, by standard results (Newey and McFadden (1994)), $\hat{\mathcal{X}}$ is a strongly consistent estimator of $\mathcal{X}^{*}$ if $\mathcal{X}^{*}$ is the unique minimizer of $D^{*}$. Indeed, $\mathcal{X}^{*}$ is a minimizer of $D^{*}$ since $D^{*}\left(\mathcal{X}^{*}\right)=0$. It turns out that it is the unique minimizer under the following identification assumption.

\footnotetext{
${ }^{18}$ All convergence statements in this paper are with respect to $N^{*} \rightarrow \infty$ where $N^{*}=\min _{A \in \mathscr{A}} N_{A}$.
} 
Assumption 1. For all subsets $A_{i} \subset X_{i}$ and $A_{j} \subseteq X_{j}$, there are menus $A, B \in \mathscr{A}$ such that $\bar{r}_{A}\left(A_{i}, A_{j}\right) \neq \bar{r}_{B}\left(A_{i}, A_{j}\right), A \cap A_{i}=B \cap A_{i}$, and $A \cap Y_{j}=B \cap Y_{j}$.

We now can state our strong consistency result.

Proposition 4. $\hat{\mathcal{X}} \stackrel{\text { a.s. }}{\longrightarrow} \mathcal{X}^{*}$ under Assumption 1 .

Proposition 4 shows that the true nest structure can be found by solving NMP. The intuition behind the result is as follows. As we see in our axiomatization, $a \sim_{p} b$ if and only if $a, b \in X_{i}$ for some $i$. Hence, IIA is satisfied between $a$ and $b$ when $a, b \in X_{i}$ and IIA is violated at least once between $a$ and $b$ when $a \in X_{i}$ and $b \in X_{j}$. Hence, the distance $\sum_{A, B \in \mathscr{A}, a, b \in A \cap B \cap Y}\left(\log \left(r_{A}(a, b)\right)-\right.$ $\left.\log \left(r_{B}(a, b)\right)\right)^{2}$ between $a$ and $b$ is smaller whenever $a, b \in X_{i}$. Hence, minimizing $D_{1}(\mathcal{Y})$ helps us to correctly identify that elements from different nests are in fact from different nests.

However, it is important to notice that $D_{1}(\mathcal{Y})$ alone is not sufficient to identify $\mathcal{X}^{*}$. For example, suppose $X=\left\{a_{1}, \ldots, a_{5}\right\}$ and $\mathcal{X}^{*}$ is given by $X_{1}=\left\{a_{1}, a_{2}, a_{3}\right\}$ and $X_{2}=\left\{a_{4}, a_{5}\right\}$. Since the data provide a noisy measure of $\bar{p}$, it is possible that $D_{1}$ is minimized at some finer partition, say $Y_{1}=\left\{a_{1}\right\}, Y_{2}=\left\{a_{2}, a_{3}\right\}$, and $Y_{3}=\left\{a_{4}, a_{5}\right\}$. Note that $\mathcal{Y}$ splits $X_{1}$, and since $D_{1}$ measures IIA violations within nests, $D_{1}(\mathcal{Y}) \leq D_{1}\left(\mathcal{X}^{*}\right)$ because $\mathcal{Y}$ never combines two elements from different nests into the same nest (i.e., it is finer than $\mathcal{X}^{*}$ ).

This example illustrates a potential problem. $D_{1}$ by itself tends to select finer partitions (it wants to create "too many nests"). The second term, $D_{2}$, corrects this problem. If $\mathcal{Y}$ were the true nest structure, our axiomatization (i.e., the second half of Independence of Symmetric Alternatives) requires that the relative likelihoods between alternatives in $Y_{2}$ (for instance, $a_{2}$ ) and alternatives in $Y_{3}$ (for instance, $a_{4}$ ) are unaffected by the presence of $a_{1}$. Accordingly, $\mathcal{Y}$ is penalized by $D_{2}$ if introducing $a_{1}$ changes the relative likelihoods between alternatives in $Y_{2}$ and $Y_{3}$. Importantly, since the true nest structure, $\mathcal{X}^{*}$, groups $a_{1}$ with $a_{2}$ and $a_{3}, \mathcal{X}^{*}$ will not be penalized, and so $D_{2}(\mathcal{Y})>D_{2}\left(\mathcal{X}^{*}\right)$ almost surely. ${ }^{19}$ Thus $D_{2}(\cdot)$ enables us to correctly conclude that $a_{1}$ and $a_{2}$ do in fact belong to the same nest.

Notice that solving NMP is quite different from the typical exercise of selecting a nest structure in the literature. In a typical nested logit estimation, a researcher assumes a nest structure and then runs a maximum likelihood (ML) estimation to identify model parameters. To compare different nest structures, the researcher has to run a full ML estimation for each nest structure. Hence, it is computationally expensive to compare many different nest structures. However, our NMP provides a data-driven way to compare different nest structures without estimating the full parametric model. Moreover, NMP does not rely on the functional form of nested logit, since it applies to any NSC.

Finally, note that $\mathcal{X}^{*}$ is a minimizer of $D$ without any further assumptions; our identifying Assumption 1 is only required to ensure that $\mathcal{X}^{*}$ is the unique minimizer. Consequently, when Assumption 1 is violated, $\mathcal{X}^{*}$ will always be contained in the set of minimizers of $D$. This suggests

\footnotetext{
${ }^{19}$ We say $Z_{n}>Z_{n}^{\prime}$ almost surely when there is $N$ such that $\operatorname{Pr}\left(Z_{n}>Z_{n}^{\prime}\right)=1$ for any $n>N$.
} 
that $D$ may still be used for nest selection and that solving NMP can facilitate identification of the true nest structure.

\subsection{Revealed Similarity and Nest Selection}

There is a practical concern with directly applying Proposition 4 to identify the nest structure because $|\mathscr{X}|$ grows exponentially as $|X|$ increases. $^{20}$ Therefore, we further refine our result by showing that we only need to compare at most $|X|$ different partitions, rather than $|\mathscr{X}|$. This dramatically reduces the number of calculations; comparing $|X|$ different partitions is computationally inexpensive even when $X$ contains hundreds of alternatives. To establish this result, we introduce the following measure of IIA violations. For any $a, b \in X$, let

$$
d(a, b) \equiv \frac{\sum_{A, B \in \mathscr{A}: a, b \in A \cap B}\left(\log \left(r_{A}(a, b)\right)-\log \left(r_{B}(a, b)\right)\right)^{2}}{|\{(A, B, a, b) \mid a, b \in A \cap B\}|} .
$$

The value of $d(a, b)$ captures the total "distance" between $a$ and $b$, in terms of IIA violations in the data $\mathcal{O}$. Consistent with our axiomatization, and the intuition behind $D_{1}$, the value of $d(a, b)$ is smaller when $a$ and $b$ are from the same nest than when they are from different nests. While conceptually similar to $D_{1}$, note that it is defined over the alternatives, not on nest structures. This crucial distinction allows us to use $d$ to narrow our candidate nests purely based on the data.

Proposition 5. Under Assumption 1, there are $\epsilon^{*}, \bar{N}>0$ such that for any $N^{*}>\bar{N}$,

$$
\max _{i} \max _{a, b \in X_{i}} d(a, b)<\epsilon^{*}<\min _{i<j} \min _{a^{\prime} \in X_{i}, b^{\prime} \in X_{j}} d\left(a^{\prime}, b^{\prime}\right) \text { with probability one. }
$$

Proposition 5 shows that for large enough $N^{*}$, there exists a "separating" threshold that correctly identifies whether two alternatives belong, or do not belong, to the same nest. If the researcher knows $\epsilon^{*}$, then identifying the nest structure is a straightforward task due to this result. But when $\epsilon^{*}$ is unknown, Proposition 5 is not sufficient to identify the nest structure. However, the insights provided by Proposition 5 allow us to show that in order to identify the correct nest structure for any NSC, only $|X|$ different partitions are worth considering. In fact, we will explicitly construct the set of partitions that need to be considered using $d$ and prove that this set contains the true nest $\mathcal{X}^{*}$.

In order to construct the set of relevant partitions, we introduce the following "approximately similar" relation: for any $\epsilon \geq 0$ and $a, b \in X$, let $a \sim_{\epsilon} b$ if $d(a, b)<\epsilon$. When $\sim_{\epsilon}$ is transitive, let $\mathcal{X}_{\epsilon} \equiv X / \sim_{\epsilon}$, which is the partition of $X$ such that for any $A \in \mathcal{X}_{\epsilon}, a \in A$, and $b \in X, a \sim_{\epsilon} b$ if and only if $b \in A$.

\footnotetext{
${ }^{20}$ Unlike the standard method of estimating nested logit, it is not computationally expensive to solve NMP by going through all possible partitions of $X$ when $|X|$ is small. For instance, there are 877 different partitions when $|X|=7$. Indeed, many papers in the literature study situations with relatively few alternatives (e.g., transportation modes or cellphone providers), and Proposition 4 can be applied to these situations directly.
} 
Since we have finite data, if $\epsilon$ and $\epsilon^{\prime}$ are close enough, they will result in the same relation $\left(\sim_{\epsilon}=\sim_{\epsilon^{\prime}}\right)$, except for certain knife-edge cases (which happens at most $|X|$ times). Notice that for smaller $\epsilon$, we are "more discriminating" in declaring similarity and this results in a finer partition. For larger $\epsilon$, we are "less discriminating" in declaring similarity and this results in a coarser partition. Let $\bar{\epsilon} \equiv \max _{a, b \in X} d(a, b)$, the maximal distance calculated in the data. Then for any $\epsilon>\bar{\epsilon}$, the resulting relation $\sim_{\epsilon}$ is complete, which reduces to the Luce model ( $\epsilon=0$ also gives the Luce model). Consequently, we never need to use an $\epsilon$ above $\bar{\epsilon}$. Because of these two key features of $\sim_{\epsilon}$, it turns out that the set $\mathscr{X}^{*} \equiv\left\{\mathcal{X}_{\epsilon}\right\}_{\epsilon \in[0, \bar{\epsilon}]}$ is the desired collection of partitions and contains at most $|X|$ different elements.

Proposition 6. $\left|\mathscr{X}^{*}\right| \leq|X|$.

Combining Propositions 4-6, we can immediately show that $\mathscr{X}^{*}$ contains the true partition structure, and it can be found by solving NMP, as formalized below. Let $\hat{\mathcal{X}}^{*}=\arg \min \mathcal{Y} \in \mathscr{X}^{*} D(\mathcal{Y})$.

Corollary 2. $\hat{\mathcal{X}}^{*} \stackrel{\text { a.s. }}{\longrightarrow} \mathcal{X}^{*}$ under Assumption 1.

The minimization problem NMP is not computationally demanding since $\left|\mathscr{X}^{*}\right| \leq|X|$. Hence, we can find the true nest structure even if there are many products. In practice, computing $\mathscr{X}^{*}$ from choice frequencies is quite simple. First note that any partition of $X$ can be represented by an $|X| \times|X|$ matrix $M$ such that $M_{x, y}=1$ when $x$ and $y$ are from the same nest and 0 otherwise. Hence, to compute $\mathscr{X}^{*}$, we follow the following steps:

1) Calculate $d(a, b)$ for each pair $(a, b)$ in $X \times X$;

2) For each $(a, b)$, construct the $|X| \times|X|$ matrix $M^{a b}$ such that $M_{x, y}^{a b}=1$ if $d(x, y)<d(a, b)$ and 0 otherwise;

3) Include the matrix $M^{a b}$ in $\mathscr{X}^{*}$ if for any $x, y, z \in X, M_{x, z}^{a b}=1$ whenever $M_{x, y}^{a b}=M_{y, z}^{a b}=1$.

The first step determines the collection of relevant thresholds from the data to construct candidate relations $\sim_{\epsilon}$. The second step generates $|X|(|X|-1) / 2$ matrices, which represent the similarity thresholds found in the previous step. The third step reduces the number to $|X|$, as we prove in Proposition 6 , since $\sim_{\epsilon}$ must be transitive.

\subsection{Identification from Simulations}

To illustrate our algorithm and our theoretical result on identification, we ran the following simulation with six alternatives. We assumed that the true nest structure is $X_{1}=\left\{x_{1}, x_{2}, x_{3}\right\}$ and $X_{2}=\left\{x_{4}, x_{5}, x_{6}\right\}$, with $X=X_{1} \cup X_{2}$, and calculated the fraction of trials in which our procedure correctly identified the nest structure. To do so, we randomly generated values for $u$ and $v$ and calculated $\bar{p}$, which is the NSC given by $\left(v, u,\left\{X_{1}, X_{2}\right\}\right)$. To introduce sampling error, we drew independent errors from a uniform distribution $U[0, \delta]$ and perturbed $\bar{p}{ }^{21}$ As shown by Proposition 4 ,

\footnotetext{
${ }^{21}$ Specifically, for each menu $A$ and each simulation trial $t$, we independently draw errors $\left\{\zeta_{a, A, t}\right\}_{a \in A}$ from $U[0, \delta]$ and construct $\bar{p}^{t}(\cdot, A)$ as follows: $\bar{p}^{t}(a, A)=\frac{\bar{p}^{t}(a, A)+\zeta_{a, A, t}}{\sum_{b \in A} \bar{p}^{t}(b, A)+\zeta_{b, A, t}}$ for each $a \in A$.
} 


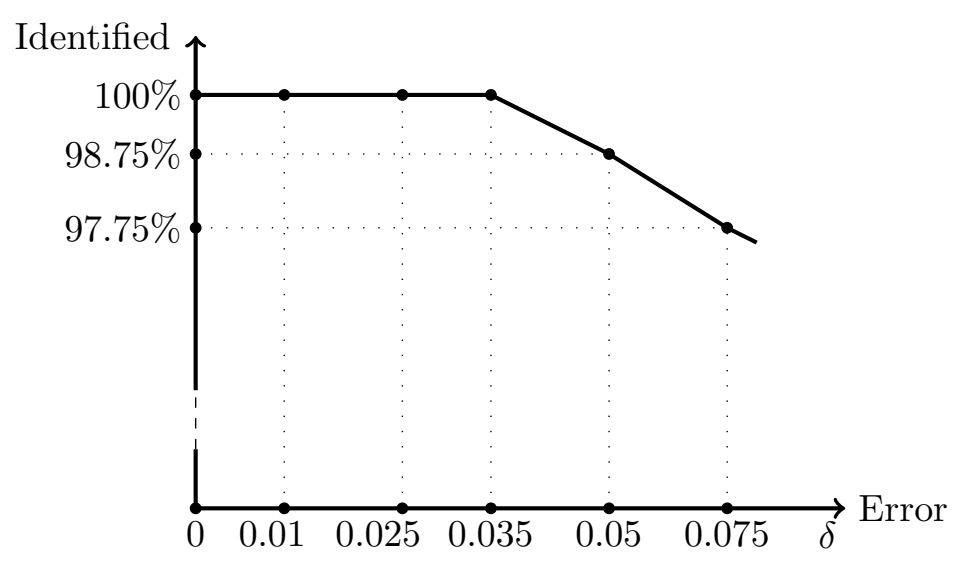

Figure 1: Percentage of trials with correctly identified nest structure with error $\delta$.

when $\delta$ is small enough, the true nest structure will be identified correctly. This was confirmed by our simulation.

We considered six different values for $\delta(\{0,0.01,0.025,0.035,0.05,0.075\})$ and ran a total of 2400 trials, the results of which are summarized in Figure 1. For $\delta \in\{0,0.01,0.025,0.035\}$, the

nest structure was correctly identified in all trials. For $\delta=0.05(0.075)$, the nest structure was correctly identified 395 (391) times out of 400 trials. In other words, in line with our theoretical result (Proposition 4), when error is relatively small (e.g., $\delta \leq 0.035$ ) the true nest is recovered $100 \%$ of the time. Even for relatively large errors (e.g., $\delta \geq 0.05$ ), we recover the true nests over $97 \%$ of the time.

\section{$6 \quad$ Related Literature}

The main contributions of our paper are the characterizations of nested logit and NSC. While nested logit is the most commonly applied model that deals with the similarity effect, many other models have been proposed. Two prominent such models are Elimination-By-Aspects (EBA) of Tversky (1972) and the Attribute Rule (AR) of Gul et al. (2014). Both EBA and AR are RUM and generalize the Luce model. While each of these models has an intersection with the NSC, neither one nests nor is nested by NSC.

In Tversky's EBA, each alternative is a collection of aspects. The decision maker randomly selects one of these aspects from the aspects available in the menu, via a Luce rule, and eliminates alternatives that do not have the selected aspect. The decision maker repeats this procedure until a single alternative remains. EBA is conceptually similar to an $N$-step nested logit, where $N$ is the total number of alternatives. The Linear NSC is a special case of EBA, but EBA is disjoint from nested logit.

In the AR of Gul et al. (2014), each alternative has many attributes. A decision maker randomly selects one attribute from the attributes available in the menu via a Luce rule. When the selected attribute is $\omega$, alternative $x$ will be chosen with a probability that is proportional to $\gamma_{x}^{\omega}$, where 
$\gamma_{x}^{\omega}$ is the intensity of $\omega$ in $x$. The AR is conceptually similar to cross-nested logit. In fact, one can show that the AR is a special case of a non-parametric version of cross-nested logit in which weights assigned to nests are menu-independent (i.e., $\gamma_{x}^{\omega}$ is menu-independent). Because of the menu independence of $\gamma_{x}^{\omega}$, the AR is more restrictive than unrestricted cross-nested logit. The Linear NSC is a special case of the AR, but nested logit is not.

Other recent papers dealing with the similarity effect are Faro (2018) and Li and Tang (2016). Both are special cases of NSC, but are generally distinct from nested logit.

Faro (2018) introduces the Luce Model with Replicas (LR), which is a special case of Linear NSC in which nest values and Luce utilities are constant: $v(A)=v_{i}$ for each $A \subseteq X_{i}$ and $u(a)=u(b)$ for any $a, b \in X_{i}$. In terms of behavior, Faro's model only allows for restrictive forms of the similarity effect in which similar alternatives are replicas.

Li and Tang (2016) present the Associationistic Luce Model (AL), which is a special case of NSC with $v(A)=\sum_{a \in A} \gamma(a)$ for some function $\gamma$. Because of the additive structure of $v$, AL is significantly more restrictive than NSC. In fact, the Luce model is the only intersection between nested logit and AL. We note that the AL also allows for violations of regularity (e.g., the attraction effect). However, since $v$ is increasing, the AL cannot simultaneously allow for violations of regularity and the similarity effect (see Appendix B.1). In terms of axiomatic foundations, they also use the revealed similarity relation $\sim_{p}$, and impose transitivity of $\sim_{p}$ as one of their axioms.

NSC has a large overlap with RUM, which goes back to Block and Marschak (1960), Falmagne (1978), and Barbera and Pattanaik (1986). For example, both random utility nested logit and Linear NSC are RUM. In addition to EBA, AR, and random utility nested logit, many special cases of RUM have been proposed, including: Gul and Pesendorfer (2006), in which each preference has an expected utility representation; Apesteguia et al. (2017), in which the collection of preferences satisfy the single-crossing property; and Manzini and Mariotti (2014), in which randomness occurs due to stochastic consideration. Our characterization of random utility nested logit contributes to this area of the stochastic choice literature.

NSC has an interpretation as a sequential choice model, in which a nest is chosen and then an alternative. Manzini and Mariotti (2012) study a deterministic choice model in which a decision maker categorizes alternatives before choosing. The decision maker first selects the "best" category according to some ordering, then selects their most preferred alternative according to another. Categories however do not need to form a partition, unlike in NSC. Ravid and Steverson (2018) introduce the following stochastic choice model that involves a sequence of binary comparisons:

$$
p(x, A)=\frac{\prod_{y \in A \backslash\{x\}} \pi(x, y)}{\sum_{z \in A} \prod_{t \in A \backslash\{z\}} \pi(z, t)} .
$$

This model, which is a special case of Marley (1991), is disjoint from nested logit but has an interesting connection to NSC. In particular, when $\pi(x, y)=\frac{1}{u(y)}$ and $\pi(x, z)=\frac{1}{w(z)}$ for any $x, y \in X_{i}$ and $z \in X_{j}$, we obtain an NSC with $v\left(A \cap X_{i}\right)=\left(\sum_{y \in A \cap X_{i}} u(y)\right) \frac{\prod_{y \in A \cap X_{i} w(y)}}{\prod_{y \in A \cap X_{i}} u(y)}$. 


\section{References}

Amemiya, T. (1985): Advanced econometrics, Harvard university press.

Anderson, S. P. And A. De Palma (1992): "Multiproduct firms: A nested logit approach," The Journal of Industrial Economics, 261-276.

Apesteguia, J., M. A. Ballester, And J. Lu (2017): "Single-Crossing Random Utility Models," Econometrica, 85, 661-674.

Barbera, S. And P. K. Pattanaik (1986): "Falmagne and the rationalizability of stochastic choices in terms of random orderings," Econometrica: Journal of the Econometric Society, 707715.

Batley, R. And S. Hess (2016): "Testing for regularity and stochastic transitivity using the structural parameter of nested logit," Transportation Research Part B, 93, 355-376.

Ben-Akiva, M. And M. Bierlaire (1999): "Discrete choice methods and their applications to short term travel decisions," in Handbook of transportation science, Springer, 5-33.

Ben-Akiva, M. E. (1973): "Structure of Passenger Travel Demand Models." Ph.D. thesis, MIT.

Bierlaire, M. (2006): "A Theoretical Analysis of the Cross-Nested Logit Model," Annals of operations Research, 144, 287-300.

Block, H. And J. Marschak (1960): Contributions to Probability and Statistics, Stanford University Press, Stanford, California, chap. Random Orderings and Stochastic Theories of Responses.

Börsch-SupAn, A. (1990): "On the compatibility of nested logit models with utility maximizatio," Journal of Econometrics, 43, 373-388.

Brownstone, D. and K. A. Small (1989): "Efficient estimation of nested logit models," Journal of Business \& Economic Statistics, 7, 67-74.

Cerreia-Vioglio, S., D. Dillenberger, P. Ortoleva, and G. Riella (2019): "Deliberately Stochastic," American Economic Review, 109.

Daly, A. And S. Zachary (1978): "Improved Multiple Choice Models," in Determinants of Travel Choice, ed. by D. A. Hensher and M. Q. Dalvi, Saxon House, 335-357.

Debreu, G. (1960): "Review of RD Luce, Individual choice behavior: A theoretical analysis," American Economic Review, 50, 186-188.

Echenique, F., K. Saito, And G. Tserenjigmid (2018): "The perception-adjusted Luce model," Mathematical Social Sciences, 93, 67-76.

Eliaz, K. AND R. Spiegler (2011): "On the strategic use of attention grabbers," Theoretical Economics, 127-155.

Falmagne, J.-C. (1978): "A representation theorem for finite random scale systems," Journal of Mathematical Psychology, 18, 52-72.

FAro, J. H. (2018): "Luce Model with Replicas," Working Paper.

Forinash, C. V. And F. S. Koppelman (1993): "Application and interpretation of nested logit models of intercity mode choice," Transportation Research Record.

Fosgerau, M., D. McFadden, And M. Bierlaire (2013): "Choice probability generating 
functions," Journal of Choice Modelling, 8, 1-18.

Foubert, B. And E. Gijsbrechts (2007): "Shopper Response to Bundle Promotions for Packaged Goods," Journal of Marketing Research, 44, 647-662.

Goldberg, P. K. (1995): "Product differentiation and oligopoly in international markets: The case of the US automobile industry," Econometrica, 891-951.

Greene, W. H. (2003): Econometric analysis, Pearson Education India.

Gul, F., P. Natenzon, and W. Pesendorfer (2014): "Random choice as behavioral optimization," Econometrica, 82, 1873-1912.

Gul, F. And W. Pesendorfer (2006): "Random Expected Utility," Econometrica, 74, 121-146.

Herriges, J. A. And C. L. Kling (1996): "Testing the consistency of nested logit models with utility maximization," Economics Letters, 50, 33-39.

Lal, R. And C. Matutes (1994): "Retail Pricing and Advertising Strategies," The Journal of Business, 67, 345-370.

LEE, B. (1999): "Calling patterns and usage of residential toll service under self selecting tariffs," Journal of Regulatory economics, 16, 45-82.

LI, J. And R. TANG (2016): "Associationistic Luce Rule," Working Paper.

Luce, R. D. (1959): Individual choice behavior: A theoretical analysis, John Wiley and sons.

Lurkin, V., L. A. Garrow, M. Higgins, J. P. Newman, and M. Schyns (2018): "Modeling competition among airline itineraries," Transportation Research Part A, 113, 157-172.

Manzini, P. And M. Mariotti (2012): "Categorize then choose: Boundedly rational choice and welfare," Journal of the European Economic Association, 10, 1141-1165.

(2014): "Stochastic choice and consideration sets," Econometrica, 82, 1153-1176.

Marley, A. (1991): "Context dependent probabilistic choice models based on measures of binary advantage," Mathematical Social Sciences, 21, 201-231.

McFAdDEn, D. (1978): "Modeling the choice of residential location," in Spatial Interaction Theory and Planning Models, ed. by A. Karlqvist, J. W. Weibull, L. Lundqvist, and F. Snickars, NorthHolland, 673, 75-96.

Natenzon, P. (2018): "Random Choice and Learning," Journal of Political Economy, forthcoming.

Newey, W. K. And D. MCFadden (1994): "Large sample estimation and hypothesis testing," Handbook of econometrics, 4, 2111-2245.

OK, E. A. (2007): Real analysis with economic applications, vol. 10, Princeton University Press.

Papola, A. (2004): "Some Developments on the cross-nested logit model," Transportation Research Part B: Methodological, 38, 833-851.

Ravid, D. And K. Steverson (2018): "Focus, Then Compare," Working Paper.

Rieskamp, J., J. R. Busemeyer, And B. A. Mellers (2006): "Extending the bounds of rationality: evidence and theories of preferential choice," Journal of Economic Literature, 631661.

Rubinstein, A. (1988): "Similarity and decision-making under risk (Is there a utility theory 
resolution to the Allais paradox?)," Journal of Economic Theory, 46, 145-153.

Shafir, S., T. A. Waite, And B. H. Smith (2002): "Context-dependent violations of rational choice in honeybees (Apis mellifera) and gray jays (Perisoreus canadensis)," Behavioral Ecology and Sociobiology, 51, 180-187.

Train, K. E. (2009): Discrete choice methods with simulation, Cambridge university press.

Train, K. E., M. Ben-Akiva, And T. Atherton (1989): "Consumption Patterns and SelfSelecting Tariffs," Review of Economics and Statistics, 71, 62-73.

Train, K. E., D. L. McFadden, and M. Ben-Akiva (1987): "The demand for local telephone service: A fully discrete model of residential calling patterns and service choices," The RAND Journal of Economics, 109-123.

Tversky, A. (1972): "Elimination by Aspects: A Theory of Choice," Psychological review.

Vovsha, P. (1997): "Application of cross-nested logit model to mode choice in Tel Aviv, Israel, metropolitan area," Transportation Research Record, 1607, 6-15.

Wen, C.-H. And F. S. Koppelman (2001): "The generalized nested logit model," Transportation Research Part B: Methodological, 35, 627-641.

\section{A Proofs}

\section{A.1 Proof of Theorem 1}

Sufficiency. We first prove the sufficiency part of Theorem 1 by the following nine steps. Suppose $p$ satisfies Independence of Symmetric Alternatives.

Step 1: $\sim_{p}$ is transitive.

Take any $x, y, z \in X$ such that $x \sim_{p} y$ and $y \sim_{p} z$. We shall prove that $\frac{p(x,\{x, z\})}{p(z,\{x, z\})}=\frac{p(x, A)}{p(z, A)}$ for any $A$ with $x, z \in A$.

Take any $A$ with $x, z \in A$ and $y \notin A$. Since $x \sim_{p} y$ and $y \sim_{p} z$, by Independence of Symmetric Alternatives we have $\frac{p(x, A \cup y)}{p(z, A \cup y)}=\frac{p(x, A)}{p(z, A)}$ and $\frac{p(x,\{x, y, z\})}{p(z,\{x, y, z\})}=\frac{p(x,\{x, z\})}{p(z,\{x, z\})}$. By the definition of $\sim_{p}, x \sim_{p} y$ implies $\frac{p(x, A \cup y)}{p(y, A \cup y)}=\frac{p(x,\{x, y, z\})}{p(y,\{x, y, z\})}$ and $y \sim_{p} z$ implies $\frac{p(y, A \cup y)}{p(z, A \cup y)}=\frac{p(y,\{x, y, z\})}{p(z,\{x, y, z\})}$. By combining all the previous equalities,

$$
\begin{aligned}
\frac{p(x, A)}{p(z, A)} & =\frac{p(x, A \cup y)}{p(z, A \cup y)}=\frac{p(x, A \cup y)}{p(y, A \cup y)} \cdot \frac{p(y, A \cup y)}{p(z, A \cup y)} \\
& =\frac{p(x,\{x, y, z\})}{p(y,\{x, y, z\})} \cdot \frac{p(y,\{x, y, z\})}{p(z,\{x, y, z\})}=\frac{p(x,\{x, y, z\})}{p(z,\{x, y, z\})}=\frac{p(x,\{x, z\})}{p(z,\{x, z\})} .
\end{aligned}
$$

Hence, $x \sim_{p} z$.

Step 2: Let $X / \sim_{p} \equiv\left\{X_{i}\right\}_{i=1}^{K}$; that is, for any $x_{i}, x_{i}^{\prime} \in X_{i}$ and $x_{j} \in X_{j}, x_{i} \sim_{p} x_{i}^{\prime}$ and $x_{i} \varkappa_{p} x_{j}$. Since $\sim_{p}$ is reflexive, transitive, and symmetric, we have a well-defined partition of $X$.

Step 3: The construction of $u$. 
Notice that for each $i \leq K$, IIA is satisfied at all subsets of $X_{i}$. Therefore, for each $i$, there is a utility function $u_{i}: X_{i} \rightarrow \mathbb{R}_{++}$such that $p(a, A)=\frac{u_{i}(a)}{\sum_{b \in A} u_{i}(b)}$ for any $A \subseteq X_{i}$ and $a \in A$ (as in the characterization of the Luce model). Since $X_{1}, \ldots, X_{k}$ are disjoint, we also have $u: X \rightarrow \mathbb{R}_{++}$ such that for any $A \subseteq X_{i}$ and $a \in A, p(a, A)=\frac{u(a)}{\sum_{b \in A} u(b)}$.

Step 4: For any $A \in \mathscr{A}$ and $a \in A \cap X_{i}$,

$$
p(a, A)=\frac{u(a)}{\sum_{x \in A \cap X_{i}} u(x)} p\left(A \cap X_{i}, A\right)
$$

Take any $A$ and $a \in A \cap X_{i}$. By the definitions of $\sim_{p}, u$, and $\left\{X_{j}\right\}_{j=1}^{K}$, we have $\frac{p\left(a^{\prime}, A\right)}{p(a, A)}=$ $\frac{p\left(a^{\prime},\left\{a^{\prime}, a\right\}\right)}{p\left(a,\left\{a^{\prime}, a\right\}\right)}=\frac{u\left(a^{\prime}\right)}{u(a)}$ for any $a^{\prime} \in A \cap X_{i}$. Then $\frac{p\left(A \cap X_{i}, A\right)}{p(a, A)}=\frac{\sum_{a^{\prime} \in A \cap X_{i}} u\left(a^{\prime}\right)}{u(a)}$. Hence, $p(a, A)=$ $\frac{u(a)}{\sum_{a^{\prime} \in A \cap X_{i}}^{u\left(a^{\prime}\right)}} p\left(A \cap X_{i}, A\right)$.

Step 5. Take alternatives $a, b, x$ such that $a \in X_{i}, b \in X_{j}$, and $x \in X_{k}$. By Independence of Symmetric Alternatives, for any $A \in \mathscr{A}$ with $a, b, x \in A$, we have $\frac{p(a, A)}{p(b, A)}=\frac{p(a, A \backslash\{x\})}{p(b, A \backslash\{x\})}$ since $a \varkappa_{p} x$ and $b \varkappa_{p} x$. Equivalently,

$$
\frac{\frac{u(a)}{\sum_{y \in A \cap X_{i}} u(y)}}{\frac{u(b)}{\sum_{z \in A \cap X_{j}} u(z)}} \cdot \frac{p\left(A \cap X_{i}, A\right)}{p\left(A \cap X_{j}, A\right)}=\frac{\frac{u(a)}{\sum_{y \in A \cap X_{i}} u(y)}}{\frac{u(b)}{\sum_{z \in A \cap X_{j}} u(z)}} \cdot \frac{p\left(A \cap X_{i}, A \backslash\{x\}\right)}{p\left(A \cap X_{j}, A \backslash\{x\}\right)} .
$$

Therefore, for any $A \in \mathscr{A}$ and $a, b, x \in A$ such that $a \in X_{i}, b \in X_{j}$, and $x \in X_{k}$,

$$
\frac{p\left(A \cap X_{i}, A\right)}{p\left(A \cap X_{j}, A\right)}=\frac{p\left(A \cap X_{i}, A \backslash\{x\}\right)}{p\left(A \cap X_{j}, A \backslash\{x\}\right)} .
$$

Step 6. We will construct the nest utility function $v: \bigcup_{i=1}^{K} 2^{X_{i}} \rightarrow \mathbb{R}_{+}$for subsets of $X_{2}, \ldots, X_{k}$ in the following way.

First, let us take $\alpha_{i} \subseteq X_{i}$ where $i \geq 2$. Let

$$
v\left(\alpha_{i}\right) \equiv \frac{p\left(\alpha_{i}, \alpha_{i} \cup X_{1}\right)}{p\left(X_{1}, \alpha_{i} \cup X_{1}\right)}
$$

Fact 1. For any $\alpha_{i} \subseteq X_{i}, \alpha_{j} \subseteq X_{j}$ with $i, j \geq 2$,

$$
\frac{p\left(\alpha_{i}, \alpha_{i} \cup \alpha_{j}\right)}{p\left(\alpha_{j}, \alpha_{i} \cup \alpha_{j}\right)}=\frac{v\left(\alpha_{i}\right)}{v\left(\alpha_{j}\right)} .
$$

Proof of Fact 1. Notice that from Equation (12) we can obtain the following by repeatedly eliminating $x \in X_{1}$ from $\alpha_{i} \cup \alpha_{j} \cup X_{1}$ :

$$
\frac{p\left(\alpha_{i}, \alpha_{i} \cup \alpha_{j} \cup X_{1}\right)}{p\left(\alpha_{j}, \alpha_{i} \cup \alpha_{j} \cup X_{1}\right)}=\frac{p\left(\alpha_{i}, \alpha_{i} \cup \alpha_{j}\right)}{p\left(\alpha_{j}, \alpha_{i} \cup \alpha_{j}\right)} .
$$


Similarly, from Equation (12) we obtain

$$
\frac{p\left(\alpha_{i}, \alpha_{i} \cup \alpha_{j} \cup X_{1}\right)}{p\left(X_{1}, \alpha_{i} \cup \alpha_{j} \cup X_{1}\right)}=\frac{p\left(\alpha_{i}, \alpha_{i} \cup X_{1}\right)}{p\left(X_{1}, \alpha_{i} \cup X_{1}\right)}=v\left(\alpha_{i}\right)
$$

and

$$
\frac{p\left(\alpha_{j}, \alpha_{i} \cup \alpha_{j} \cup X_{1}\right)}{p\left(X_{1}, \alpha_{i} \cup \alpha_{j} \cup X_{1}\right)}=\frac{p\left(\alpha_{j}, \alpha_{j} \cup X_{1}\right)}{p\left(X_{1}, \alpha_{j} \cup X_{1}\right)}=v\left(\alpha_{j}\right) .
$$

Combining the above three equalities, we obtain

$$
\frac{v\left(\alpha_{i}\right)}{v\left(\alpha_{j}\right)}=\frac{\frac{p\left(\alpha_{i}, \alpha_{i} \cup \alpha_{j} \cup X_{1}\right)}{p\left(X_{1}, \alpha_{i} \cup \alpha_{j} \cup X_{1}\right)}}{\frac{p\left(\alpha_{j}, \alpha_{i} \cup \alpha_{j} \cup X_{1}\right)}{p\left(X_{1}, \alpha_{i} \cup \alpha_{j} \cup X_{1}\right)}}=\frac{p\left(\alpha_{i}, \alpha_{i} \cup \alpha_{j} \cup X_{1}\right)}{p\left(\alpha_{j}, \alpha_{i} \cup \alpha_{j} \cup X_{1}\right)}=\frac{p\left(\alpha_{i}, \alpha_{i} \cup \alpha_{j}\right)}{p\left(\alpha_{j}, \alpha_{i} \cup \alpha_{j}\right)}
$$

Fact 2. For any $\alpha_{i} \subseteq X_{i}, \alpha_{j} \subseteq X_{j}$ with $i, j \geq 2$ and $A \subseteq \cup_{s \neq i, j} X_{s}$,

$$
\frac{p\left(\alpha_{i}, \alpha_{i} \cup \alpha_{j} \cup A\right)}{p\left(\alpha_{j}, \alpha_{i} \cup \alpha_{j} \cup A\right)}=\frac{v\left(\alpha_{i}\right)}{v\left(\alpha_{j}\right)} .
$$

Proof of Fact 2. By Equation (12), we obtain the following equality by repeatedly eliminating $x \in A$ from $\alpha_{i} \cup \alpha_{j} \cup A$ :

$$
\frac{p\left(\alpha_{i}, \alpha_{i} \cup \alpha_{j} \cup A\right)}{p\left(\alpha_{j}, \alpha_{i} \cup \alpha_{j} \cup A\right)}=\frac{p\left(\alpha_{i}, \alpha_{i} \cup \alpha_{j}\right)}{p\left(\alpha_{j}, \alpha_{i} \cup \alpha_{j}\right)}=\frac{v\left(\alpha_{i}\right)}{v\left(\alpha_{j}\right)} .
$$

Step 7. We will construct the nest utility function $v: \bigcup_{i=1}^{K} 2^{X_{i}} \rightarrow \mathbb{R}_{+}$for subsets of $X_{1}$ in the following way.

First, let us take $\alpha_{1} \subseteq X_{1}$. Let

$$
v\left(\alpha_{1}\right) \equiv \frac{p\left(\alpha_{1}, \alpha_{1} \cup X_{2}\right)}{p\left(X_{2}, \alpha_{1} \cup X_{2}\right)} \cdot \frac{p\left(X_{2}, X_{1} \cup X_{2}\right)}{p\left(X_{1}, X_{1} \cup X_{2}\right)} .
$$

Fact 3. For any $\alpha_{2} \subseteq X_{2}$,

$$
\frac{p\left(\alpha_{1}, \alpha_{1} \cup \alpha_{2}\right)}{p\left(\alpha_{2}, \alpha_{1} \cup \alpha_{2}\right)}=\frac{v\left(\alpha_{1}\right)}{v\left(\alpha_{2}\right)}
$$

Proof of Fact 3. Since $v\left(\alpha_{2}\right)=\frac{p\left(\alpha_{2}, X_{1} \cup \alpha_{2}\right)}{p\left(X_{1}, X_{1} \cup \alpha_{2}\right)}$, we shall prove that

$$
\frac{p\left(\alpha_{1}, \alpha_{1} \cup X_{2}\right)}{p\left(X_{2}, \alpha_{1} \cup X_{2}\right)} \cdot \frac{p\left(X_{2}, X_{1} \cup X_{2}\right)}{p\left(X_{1}, X_{1} \cup X_{2}\right)}=\frac{p\left(\alpha_{1}, \alpha_{1} \cup \alpha_{2}\right)}{p\left(\alpha_{2}, \alpha_{1} \cup \alpha_{2}\right)} \cdot \frac{p\left(\alpha_{2}, X_{1} \cup \alpha_{2}\right)}{p\left(X_{1}, X_{1} \cup \alpha_{2}\right)} .
$$

Notice that from Equation (12) we can obtain the following equalities by repeatedly eliminating $x \in X_{3}$ from $\alpha_{1} \cup X_{2} \cup X_{3}$ and $\alpha_{1} \cup \alpha_{2} \cup X_{3}$,

$$
\frac{p\left(\alpha_{1}, \alpha_{1} \cup X_{2}\right)}{p\left(X_{2}, \alpha_{1} \cup X_{2}\right)} \cdot \frac{p\left(X_{2}, X_{1} \cup X_{2}\right)}{p\left(X_{1}, X_{1} \cup X_{2}\right)}=\frac{p\left(\alpha_{1}, \alpha_{1} \cup X_{2} \cup X_{3}\right)}{p\left(X_{2}, \alpha_{1} \cup X_{2} \cup X_{3}\right)} \cdot \frac{p\left(X_{2}, X_{1} \cup X_{2}\right)}{p\left(X_{1}, X_{1} \cup X_{2}\right)}
$$


and

$$
\frac{p\left(\alpha_{1}, \alpha_{1} \cup \alpha_{2}\right)}{p\left(\alpha_{2}, \alpha_{1} \cup \alpha_{2}\right)} \cdot \frac{p\left(\alpha_{2}, X_{1} \cup \alpha_{2}\right)}{p\left(X_{1}, X_{1} \cup \alpha_{2}\right)}=\frac{p\left(\alpha_{1}, \alpha_{1} \cup \alpha_{2} \cup X_{3}\right)}{p\left(\alpha_{2}, \alpha_{1} \cup \alpha_{2} \cup X_{3}\right)} \cdot \frac{p\left(\alpha_{2}, X_{1} \cup \alpha_{2}\right)}{p\left(X_{1}, X_{1} \cup \alpha_{2}\right)} .
$$

Therefore, we shall prove that

$$
\frac{p\left(\alpha_{1}, \alpha_{1} \cup X_{2} \cup X_{3}\right)}{p\left(X_{2}, \alpha_{1} \cup X_{2} \cup X_{3}\right)} \cdot \frac{p\left(X_{2}, X_{1} \cup X_{2}\right)}{p\left(X_{1}, X_{1} \cup X_{2}\right)}=\frac{p\left(\alpha_{1}, \alpha_{1} \cup \alpha_{2} \cup X_{3}\right)}{p\left(\alpha_{2}, \alpha_{1} \cup \alpha_{2} \cup X_{3}\right)} \cdot \frac{p\left(\alpha_{2}, X_{1} \cup \alpha_{2}\right)}{p\left(X_{1}, X_{1} \cup \alpha_{2}\right)} .
$$

Moreover,

$$
\begin{aligned}
\frac{p\left(\alpha_{1}, \alpha_{1} \cup X_{2} \cup X_{3}\right)}{p\left(X_{2}, \alpha_{1} \cup X_{2} \cup X_{3}\right)} \cdot \frac{p\left(X_{2}, X_{1} \cup X_{2}\right)}{p\left(X_{1}, X_{1} \cup X_{2}\right)} & =\frac{p\left(\alpha_{1}, \alpha_{1} \cup X_{2} \cup X_{3}\right)}{p\left(X_{3}, \alpha_{1} \cup X_{2} \cup X_{3}\right)} \cdot \frac{p\left(X_{3}, \alpha_{1} \cup X_{2} \cup X_{3}\right)}{p\left(X_{2}, \alpha_{1} \cup X_{2} \cup X_{3}\right)} \cdot \frac{p\left(X_{2}, X_{1} \cup X_{2}\right)}{p\left(X_{1}, X_{1} \cup X_{2}\right)} \\
& =\frac{p\left(\alpha_{1}, \alpha_{1} \cup X_{2} \cup X_{3}\right)}{p\left(X_{3}, \alpha_{1} \cup X_{2} \cup X_{3}\right)} \cdot \frac{v\left(X_{3}\right)}{v\left(X_{2}\right)} \cdot \frac{p\left(X_{2}, X_{1} \cup X_{2}\right)}{p\left(X_{1}, X_{1} \cup X_{2}\right)}, \text { by Fact } 2, \\
& =\frac{p\left(\alpha_{1}, \alpha_{1} \cup X_{2} \cup X_{3}\right)}{p\left(X_{3}, \alpha_{1} \cup X_{2} \cup X_{3}\right)} \cdot \frac{v\left(X_{3}\right)}{v\left(X_{2}\right)} \cdot v\left(X_{2}\right), \text { by the definition of } v,
\end{aligned}
$$

and

$$
\begin{aligned}
\frac{p\left(\alpha_{1}, \alpha_{1} \cup \alpha_{2} \cup X_{3}\right)}{p\left(\alpha_{2}, \alpha_{1} \cup \alpha_{2} \cup X_{3}\right)} \cdot \frac{p\left(\alpha_{2}, X_{1} \cup \alpha_{2}\right)}{p\left(X_{1}, X_{1} \cup \alpha_{2}\right)} & =\frac{p\left(\alpha_{1}, \alpha_{1} \cup \alpha_{2} \cup X_{3}\right)}{p\left(X_{3}, \alpha_{1} \cup \alpha_{2} \cup X_{3}\right)} \cdot \frac{p\left(X_{3}, \alpha_{1} \cup \alpha_{2} \cup X_{3}\right)}{p\left(\alpha_{2}, \alpha_{1} \cup \alpha_{2} \cup X_{3}\right)} \cdot \frac{p\left(\alpha_{2}, X_{1} \cup \alpha_{2}\right)}{p\left(X_{1}, X_{1} \cup \alpha_{2}\right)} \\
& =\frac{p\left(\alpha_{1}, \alpha_{1} \cup \alpha_{2} \cup X_{3}\right)}{p\left(X_{3}, \alpha_{1} \cup \alpha_{2} \cup X_{3}\right)} \cdot \frac{v\left(X_{3}\right)}{v\left(\alpha_{2}\right)} \cdot \frac{p\left(\alpha_{2}, X_{1} \cup \alpha_{2}\right)}{p\left(X_{1}, X_{1} \cup \alpha_{2}\right)}, \text { by Fact 2, } \\
& =\frac{p\left(\alpha_{1}, \alpha_{1} \cup \alpha_{2} \cup X_{3}\right)}{p\left(X_{3}, \alpha_{1} \cup \alpha_{2} \cup X_{3}\right)} \cdot \frac{v\left(X_{3}\right)}{v\left(\alpha_{2}\right)} \cdot v\left(\alpha_{2}\right), \text { by the definition of } v .
\end{aligned}
$$

Finally, we shall prove that

$$
\frac{p\left(\alpha_{1}, \alpha_{1} \cup X_{2} \cup X_{3}\right)}{p\left(X_{3}, \alpha_{1} \cup X_{2} \cup X_{3}\right)}=\frac{p\left(\alpha_{1}, \alpha_{1} \cup \alpha_{2} \cup X_{3}\right)}{p\left(X_{3}, \alpha_{1} \cup \alpha_{2} \cup X_{3}\right)},
$$

which immediately follows from Equation (12) by repeatedly eliminating $x \in X_{2} \backslash \alpha_{2}$ from $\alpha_{1} \cup$ $X_{2} \cup X_{3}$.

Fact 4. For any $\alpha_{i} \subseteq X_{i}$ with $i \geq 3$,

$$
\frac{p\left(\alpha_{1}, \alpha_{1} \cup \alpha_{i}\right)}{p\left(\alpha_{i}, \alpha_{1} \cup \alpha_{i}\right)}=\frac{v\left(\alpha_{1}\right)}{v\left(\alpha_{i}\right)} .
$$

Proof of Fact 4. By Equation (12) and Facts 2-3,

$$
\begin{aligned}
\frac{p\left(\alpha_{1}, \alpha_{1} \cup \alpha_{i}\right)}{p\left(\alpha_{i}, \alpha_{1} \cup \alpha_{i}\right)} & =\frac{p\left(\alpha_{1}, \alpha_{1} \cup \alpha_{i} \cup X_{2}\right)}{p\left(\alpha_{i}, \alpha_{1} \cup \alpha_{i} \cup X_{2}\right)}=\frac{p\left(\alpha_{1}, \alpha_{1} \cup \alpha_{i} \cup X_{2}\right)}{p\left(X_{2}, \alpha_{1} \cup \alpha_{i} \cup X_{2}\right)} \cdot \frac{p\left(X_{2}, \alpha_{1} \cup \alpha_{i} \cup X_{2}\right)}{p\left(\alpha_{i}, \alpha_{1} \cup \alpha_{i} \cup X_{2}\right)} \\
& =\frac{p\left(\alpha_{1}, \alpha_{1} \cup X_{2}\right)}{p\left(X_{2}, \alpha_{1} \cup X_{2}\right)} \cdot \frac{v\left(X_{2}\right)}{v\left(\alpha_{i}\right)}=\frac{v\left(\alpha_{1}\right)}{v\left(X_{2}\right)} \cdot \frac{v\left(X_{2}\right)}{v\left(\alpha_{i}\right)}=\frac{v\left(\alpha_{1}\right)}{v\left(\alpha_{i}\right)} .
\end{aligned}
$$

Fact 5. For any $\alpha_{i} \subseteq X_{i}$ with $i \geq 2$ and $A \subseteq \cup_{s \neq i, 1} X_{s}$, 


$$
\frac{p\left(\alpha_{1}, \alpha_{1} \cup \alpha_{i} \cup A\right)}{p\left(\alpha_{i}, \alpha_{1} \cup \alpha_{i} \cup A\right)}=\frac{v\left(\alpha_{1}\right)}{v\left(\alpha_{i}\right)} .
$$

Proof of Fact 5. From Equation (12), we have

$$
\frac{p\left(\alpha_{1}, \alpha_{1} \cup \alpha_{i} \cup A\right)}{p\left(\alpha_{i}, \alpha_{1} \cup \alpha_{i} \cup A\right)}=\frac{p\left(\alpha_{1}, \alpha_{1} \cup \alpha_{i}\right)}{p\left(\alpha_{i}, \alpha_{1} \cup \alpha_{i}\right)}=\frac{v\left(\alpha_{1}\right)}{v\left(\alpha_{i}\right)} .
$$

Step 8. By Facts 2, 4, and 5, for any $A \in \mathscr{A}$ and $i, j \leq K$,

$$
\frac{p\left(A \cap X_{i}, A\right)}{p\left(A \cap X_{j}, A\right)}=\frac{v\left(A \cap X_{i}\right)}{v\left(A \cap X_{j}\right)}
$$

Since $\sum_{i=1}^{K} p\left(A \cap X_{i}, A\right)=1$, we have $p\left(A \cap X_{i}, A\right)=\frac{v\left(A \cap X_{i}\right)}{\sum_{j=1}^{K} v\left(A \cap X_{j}\right)}$. Since $\frac{p(a, A)}{p(b, A)}=\frac{u(a)}{u(b)}$ for any $a, b \in X_{i}$, we have

$$
p\left(a_{i}, A\right)=\frac{u\left(a_{i}\right)}{\sum_{x \in A \cap X_{i}} u(x)} \cdot \frac{v\left(A \cap X_{i}\right)}{\sum_{j=1}^{K} v\left(A \cap X_{j}\right)} \text { for each } a_{i} \in A \cap X_{i} .
$$

Step 9. An NSC $p$ is nondegenerate.

By way of contradiction, suppose there are $i, j \leq K$ such that for some $a \in X_{i}$ and $b \in X_{j}$,

$$
\frac{\sum_{x \in A_{i}} u(x)}{v\left(A_{i}\right)}=\frac{u(a)}{v(a)} \text { and } \frac{\sum_{y \in A_{j}} u(y)}{v\left(A_{j}\right)}=\frac{u(b)}{v(b)} \text { for any } A_{i} \subseteq X_{i} \text { and } A_{j} \subseteq X_{j} \text {. }
$$

In other words,

$$
\frac{\sum_{x \in A_{i}} u(x)}{v\left(A_{i}\right)} / \frac{u(a)}{v(a)}=\frac{\sum_{y \in A_{j}} u(y)}{v\left(A_{j}\right)} / \frac{u(b)}{v(b)} \text { for any } A_{i} \subseteq X_{i} \text { and } A_{j} \subseteq X_{j} .
$$

Then by NSC representation, we have $\frac{p(a,\{a, b\})}{p(b,\{a, b\})}=\frac{p(a, A)}{p(b, A)}$ for any $A \in \mathscr{A}$; i.e., $a \sim_{p} b$, which contradicts the construction of $\left\{X_{k}\right\}_{k=1}^{K}$.

Necessity. Suppose $p$ is a nondegenerate NSC with $\left(v, u,\left\{X_{i}\right\}_{i=1}^{K}\right)$.

Step 1. For any $a, b \in X, a \sim_{p} b$ if and only if either $a, b \in X_{i}$ for some $i$.

Take any $a, b \in X$. We consider two cases.

Case 1. Suppose $a, b \in X_{i}$.

In this case, by NSC representation, $\frac{p(a, A)}{p(b, A)}=\frac{u(a)}{u(b)}=\frac{p(a,\{a, b\})}{p(b,\{a, b\})}$ for any $A \in \mathscr{A}$. Therefore, $a \sim_{p} b$.

Case 2. Suppose $a \in X_{i}$ and $b \in X_{j}$ with $i \neq j$.

We shall prove that $a \varkappa_{p} b$. By nondegeneracy of $p$, either

$$
\frac{\sum_{x \in A_{i}} u(x)}{v\left(A_{i}\right)} \neq \frac{u(a)}{v(a)} \text { for some } A_{i} \subseteq X_{i} \text { with } a \in A_{i}
$$


or

$$
\frac{\sum_{y \in A_{j}} u(y)}{v\left(A_{j}\right)} \neq \frac{u(b)}{v(b)} \text { for some } A_{j} \subseteq X_{j} \text { wtih } b \in A_{j} .
$$

Without loss of generality, suppose the former is true. Then we have

$$
\frac{p(a,\{a, b\})}{p(b,\{a, b\})}=\frac{v(a)}{v(b)} \neq \frac{p\left(a, A_{i} \cup b\right)}{p\left(b, A_{i} \cup b\right)}=\frac{u(a)}{\sum_{x \in A_{i}} u(x)} \frac{v\left(A_{i}\right)}{v(b)} .
$$

Therefore, $a \chi_{p} b$.

Step 2. The first part of Independence of Symmetric Alternatives is satisfied.

Take any $A \in \mathscr{A}, a, b \in A$, and $x \notin A$ such that $a \sim_{p} x$ and $b \sim_{p} x$. By Step 1 , we have $a, b, x \in X_{i}$ for some $i$. Therefore, $a \sim_{p} b$ implies $\frac{p(a, A)}{p(b, A)}=\frac{p(a,\{a, b\})}{p(b,\{a, b\})}=\frac{p(a, A \cup x)}{p(b, A \cup x)}$.

Step 3. The second part of Independence of Symmetric Alternatives is satisfied.

Take any $A \in \mathscr{A}, a, b \in A$, and $x \notin A$ such that $a \chi_{p} x$ and $b \varkappa_{p} x$. By Step $1, a \chi_{p} x$ and $b \nsim_{p} x$ imply $x \in X_{i}$ and $a, b \notin X_{i}$ for some $i$.

Case 1. $a, b \in X_{j}$ for some $j$.

Since $a \sim_{p} b$,

$$
\frac{p(a, A)}{p(b, A)}=\frac{p(a,\{a, b\})}{p(b,\{a, b\})}=\frac{p(a, A \cup x)}{p(b, A \cup x)} .
$$

Case 2. $a \in X_{j}$ and $b \in X_{k}$ for some $j, k$ with $j \neq k$.

In this case, we have

$$
\frac{p(a, A)}{p(b, A)}=\frac{\frac{u(a)}{\sum_{y \in A_{j}} u(y)} v\left(A_{j}\right)}{\frac{u(b)}{\sum_{z \in A_{k}} u(z)} v\left(A_{k}\right)}=\frac{p(a, A \cup x)}{p(b, A \cup x)}
$$

since $A_{j}=(A \cup x) \cap X_{j}=A \cap X_{j}$ and $A_{k}=(A \cup x) \cap X_{k}=A \cap X_{k}$.

\section{A.2 Proof of Proposition 1}

Since the uniqueness of Luce utilities and nest utilities follows standard arguments, we only prove that the nest structure is unique. By way of contradiction, suppose $p$ is a nondegenerate NSC with respect to both of $\left(v, u,\left\{X_{i}\right\}_{i=1}^{K}\right)$ and $\left(v^{\prime}, u^{\prime},\left\{X_{i}^{\prime}\right\}_{i=1}^{K^{\prime}}\right)$, and $\left\{X_{i}^{\prime}\right\}_{i=1}^{K^{\prime}}$ is not a permutation of $\left\{X_{i}\right\}_{i=1}^{K}$. Without loss of generality, suppose $K^{\prime} \geq K$. Then there are $x_{i}, x_{i}^{\prime} \in X_{i}$ such that $x_{i} \in X_{j}^{\prime}$ and $x_{i}^{\prime} \in X_{k}^{\prime}$. Since $\frac{p\left(x_{i},\left\{x_{i}, x_{i}^{\prime}\right\}\right)}{p\left(x_{i}^{\prime},\left\{x_{i}, x_{i}^{\prime}\right\}\right)}=\frac{p\left(x_{i}, A\right)}{p\left(x_{i}^{\prime}, A\right)}$ for any $A$, by NSC representation with $\left\{v^{\prime}, u^{\prime},\left\{X_{i}^{\prime}\right\}_{i=1}^{K^{\prime}}\right\}$, we have

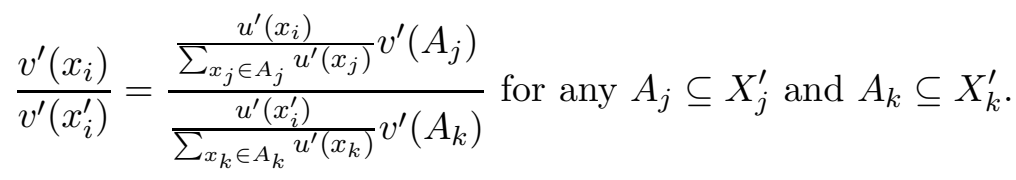


Let us first set $A_{j}=\left\{x_{i}\right\}$. Then we have $\frac{u^{\prime}\left(x_{i}^{\prime}\right)}{v^{\prime}\left(x_{i}^{\prime}\right)}=\frac{\sum_{x_{k} \in A_{k}} u^{\prime}\left(x_{k}\right)}{v^{\prime}\left(A_{k}\right)}$ for any $A_{k} \subseteq X_{k}^{\prime}$. Similarly, by setting $A_{k}=\left\{x_{i}^{\prime}\right\}$, we also obtain $\frac{u^{\prime}\left(x_{i}\right)}{v^{\prime}\left(x_{i}\right)}=\frac{\sum_{x_{j} \in A_{j}} u^{\prime}\left(x_{j}\right)}{v^{\prime}\left(A_{j}\right)}$ for any $A_{j} \subseteq X_{j}^{\prime}$. Therefore, we obtain a contradiction since the above two equalities contradict the assumption that NSC $p$ with $\left(v^{\prime}, u^{\prime},\left\{X_{i}^{\prime}\right\}_{i=1}^{K^{\prime}}\right)$ is nondegenerate.

\section{A.3 Proof of Theorem 2}

Since the necessity part is straightforward, we only prove the sufficiency part. Suppose $p$ is an NSC with $\left(v, u,\left\{X_{i}\right\}_{i=1}^{K}\right)$ and that it satisfies Log Ratio Invariance. If $K=1$, then we immediately have a Luce model. Suppose now $K \geq 2$. For each $i \leq K$, fix some $a_{i}^{*} \in X_{i}$. Then define

$$
\eta_{i} \equiv \frac{\log \left(v\left(X_{i}\right) / v\left(\left\{a_{i}^{*}\right\}\right)\right)}{\log \left(\sum_{a_{i} \in X_{i}} u\left(a_{i}\right) / u\left(a_{i}^{*}\right)\right)} .
$$

For any $A \subset X_{i}$ and any $x \in X \backslash X_{i}$, by Log Ratio Invariance, we have

$$
\frac{\log \left(\frac{p\left(X_{i}, X_{i} \cup x\right)}{p\left(x, X_{i} \cup x\right)} / \frac{p\left(a_{i}^{*},\left\{a_{i}^{*}, x\right\}\right)}{p\left(x,\left\{a_{i}^{*}, x\right\}\right)}\right)}{\log \left(\frac{p\left(X_{i}, X_{i} \cup a_{i}^{*}\right)}{p\left(a_{i}^{*}, X \cup a_{i}^{*}\right)}\right)}=\frac{\log \left(\frac{p(A, A \cup x)}{p(x, A \cup x)} / \frac{p\left(a_{i}^{*},\left\{a_{i}^{*}, x\right\}\right)}{p\left(x,\left\{a_{i}^{*}, x\right\}\right)}\right)}{\log \left(\frac{p\left(A, A \cup a_{i}^{*}\right)}{p\left(a_{i}^{*}, A \cup a_{i}^{*}\right)}\right)} .
$$

Since

$$
\frac{\log \left(\frac{p\left(X_{i}, X_{i} \cup x\right)}{p\left(x, X_{i} \cup x\right)} / \frac{p\left(a_{i}^{*},\left\{a_{i}^{*}, x\right\}\right)}{p\left(x,\left\{a_{i}^{*}, x\right\}\right)}\right)}{\log \left(\frac{p\left(X_{i}, X_{i} \cup a_{i}^{*}\right)}{p\left(a_{i}^{*}, X \cup a_{i}^{*}\right)}\right)}=\frac{\log \left(\frac{v\left(X_{i}\right)}{v\left(\left\{a_{i}^{*}\right\}\right)}\right)}{\log \left(\frac{\sum_{b \in X_{i}} u(b)}{u\left(a_{i}^{*}\right)}\right)}=\eta_{i},
$$

we have

$$
\eta_{i}=\frac{\log \left(\frac{p(A, A \cup x)}{p(x, A \cup x)} / \frac{p\left(a_{i}^{*},\left\{a_{i}^{*}, x\right\}\right)}{p\left(x,\left\{a_{i}^{*}, x\right\}\right)}\right)}{\log \left(\frac{p\left(A, A \cup a_{i}^{*}\right)}{p\left(a_{i}^{*}, A \cup a_{i}^{*}\right)}\right)}=\frac{\log \left(\frac{v(A)}{v\left(\left\{a_{i}^{*}\right\}\right)}\right)}{\log \left(\frac{\sum_{b \in A} u(b)}{u\left(a_{i}^{*}\right)}\right)}
$$

equivalently, $\frac{v(A)}{v\left(\left\{a_{i}^{*}\right\}\right)}=\left(\frac{\sum_{b \in A} u(b)}{u\left(a_{i}^{*}\right)}\right)^{\eta_{i}}$. Let $\delta_{i}=\frac{v\left(\left\{a_{i}^{*}\right\}\right)}{\left(u\left(a_{i}^{*}\right)\right)^{\eta_{i}}}$. Then $v(A)=\delta_{i}\left(\sum_{b \in A} u(b)\right)^{\eta_{i}}$. Therefore, $p$ is the nested logit with $\left(\eta_{1}, \ldots, \eta_{K}, u^{\prime},\left\{X_{i}\right\}_{i=1}^{K}\right)$ such that $u^{\prime}(x)=\delta_{i}^{\frac{1}{\eta_{i}}} u(x)$ when $x \in X_{i}$.

\section{A.4 Proof of Proposition 2}

Suppose $p$ is a nested logit with $\left(\eta_{1}, \ldots, \eta_{K}, u,\left\{X_{i}\right\}_{i=1}^{K}\right)$. Take any $A, A^{\prime} \in \mathscr{A}$ such that IIA is satisfied at $A \cup A^{\prime}$. We shall prove that Relative Likelihood Independence is satisfied. Since $p$ is a nondegenerate NSC, by Step 1 of the necessity part proof of Theorem $1, x \sim_{p} y$ if and only if $x, y \in X_{i}$ for some $i \leq K$. Take any $A, B, A^{\prime}, B^{\prime} \in \mathscr{A}$ and $x \in X$ such that $a \sim_{p} a^{\prime}$ for any $a, a^{\prime} \in A \cup B \cup A^{\prime} \cup B^{\prime}$. By the previous argument, $A \cup B \cup A^{\prime} \cup B^{\prime} \subseteq X_{i}$. By the nested logit representation,

$$
\frac{p(A, A \cup B)}{p(B, A \cup B)}=\frac{\sum_{a \in A} u(a)}{\sum_{b \in B} u(b)} \geq \frac{p\left(A^{\prime}, A^{\prime} \cup B^{\prime}\right)}{p\left(B^{\prime}, A^{\prime} \cup B^{\prime}\right)}=\frac{\sum_{a \in A^{\prime}} u(a)}{\sum_{b \in B^{\prime}} u(b)} .
$$


When $x \in X_{i}$,

$$
\frac{p(A, A \cup x)}{p(x, A \cup x)} / \frac{p(B, B \cup x)}{p(x, B \cup x)}=\frac{\sum_{a \in A} u(a)}{\sum_{b \in B} u(b)} \geq \frac{p\left(A^{\prime}, A^{\prime} \cup x\right)}{p\left(x, A^{\prime} \cup x\right)} / \frac{p\left(B^{\prime}, B^{\prime} \cup x\right)}{p\left(x, B^{\prime} \cup x\right)}=\frac{\sum_{a \in A^{\prime}} u(a)}{\sum_{b \in B^{\prime}} u(b)} .
$$

When $x \in X_{j}$ and $i \neq j$,

$$
\frac{p(A, A \cup x)}{p(x, A \cup x)} / \frac{p(B, B \cup x)}{p(x, B \cup x)}=\frac{\left(\sum_{a \in A} u(a)\right)^{\eta_{i}}}{\left(\sum_{b \in B} u(b)\right)^{\eta_{i}}} \geq \frac{p\left(A^{\prime}, A^{\prime} \cup x\right)}{p\left(x, A^{\prime} \cup x\right)} / \frac{p\left(B^{\prime}, B^{\prime} \cup x\right)}{p\left(x, B^{\prime} \cup x\right)}=\frac{\left(\sum_{a \in A^{\prime}} u(a)\right)^{\eta_{i}}}{\left(\sum_{b \in B^{\prime}} u(b)\right)^{\eta_{i}}} .
$$

Hence,

$$
\frac{p(A, A \cup B)}{p(B, A \cup B)} \geq \frac{p\left(A^{\prime}, A^{\prime} \cup B^{\prime}\right)}{p\left(B^{\prime}, A^{\prime} \cup B^{\prime}\right)} \text { implies } \frac{p(A, A \cup x)}{p(x, A \cup x)} / \frac{p(B, B \cup x)}{p(x, B \cup x)} \geq \frac{p\left(A^{\prime}, A^{\prime} \cup x\right)}{p\left(x, A^{\prime} \cup x\right)} / \frac{p\left(B^{\prime}, B^{\prime} \cup x\right)}{p\left(x, B^{\prime} \cup x\right)} .
$$

Suppose $p$ is the nondegenerate NSC with $\left(v, u,\left\{X_{i}\right\}_{i=1}^{K}\right)$ and satisfies Relative Likelihood Independence. If $K=1$, we trivially obtain the desired result. Suppose $K \geq 2$. Take any $i, j \leq K$ with $i \neq j$. Take any $A, B, A^{\prime} \subseteq X_{i}$ and $x \in X_{j}$. By Relative Likelihood Independence,

$$
\frac{p(A, A \cup B)}{p(B, A \cup B)} \geq 1 \text { implies } \frac{p(A, A \cup x)}{p(x, A \cup x)} / \frac{p(B, B \cup x)}{p(x, B \cup x)} \geq 1
$$

equivalently,

$$
p(A, A \cup B) \geq p(B, A \cup B) \text { implies } \frac{p(A, A \cup x)}{p(x, A \cup x)} \geq \frac{p(B, B \cup x)}{p(x, B \cup x)} .
$$

By the NSC representation, $\sum_{a \in A} u(a) \geq \sum_{b \in B} u(b)$ implies

$$
\frac{p(A, A \cup x)}{p(x, A \cup x)}=\frac{v(A)}{v(x)} \geq \frac{p(B, B \cup x)}{p(x, B \cup x)}=\frac{v(B)}{v(x)} .
$$

Then $\sum_{a \in A} u(a) \geq \sum_{b \in B} u(b)$ implies $v(A) \geq v(B)$. Therefore, there is an increasing function $f_{i}: \mathbb{R}_{++} \rightarrow \mathbb{R}_{++}$such that $v(A)=f_{i}\left(\sum_{a \in A} u(a)\right)$ for any $A \subseteq X_{i}$.

\section{A.5 Proof of Theorem 3}

Suppose $p$ is the nondegenerate NSC with $\left(v, u,\left\{X_{i}\right\}_{i=1}^{K}\right)$ and satisfies Relative Likelihood Independence and Richness. We shall show that $p$ is a nested logit. If $K=1$, we trivially obtain the desired result. Suppose $K \geq 2$. Let us fix $i \leq K$.

Take any $j \leq K$ with $i \neq j$. Take any $A, B, A^{\prime}, B^{\prime} \subseteq X_{i}$ and $x \in X_{j}$. By Relative Likelihood Independence and the NSC representation,

$$
\begin{gathered}
\frac{p(A, A \cup B)}{p(B, A \cup B)}=\frac{\sum_{a \in A} u(a)}{\sum_{b \in B} u(b)} \geq \frac{p\left(A^{\prime}, A^{\prime} \cup B^{\prime}\right)}{p\left(B^{\prime}, A^{\prime} \cup B^{\prime}\right)}=\frac{\sum_{a \in A^{\prime}} u(a)}{\sum_{b \in B^{\prime}} u(b)} \\
\text { implies } \frac{p(A, A \cup x)}{p(x, A \cup x)} / \frac{p(B, B \cup x)}{p(x, B \cup x)}=\frac{v(A)}{v(B)} \geq \frac{p\left(A^{\prime}, A^{\prime} \cup x\right)}{p\left(x, A^{\prime} \cup x\right)} / \frac{p\left(B^{\prime}, B^{\prime} \cup x\right)}{p\left(x, B^{\prime} \cup x\right)}=\frac{v\left(A^{\prime}\right)}{v\left(B^{\prime}\right)} .
\end{gathered}
$$


Equivalently,

$$
\frac{\sum_{a \in A} u(a)}{\sum_{b \in B} u(b)} \geq \frac{\sum_{a \in A^{\prime}} u(a)}{\sum_{b \in B^{\prime}} u(b)} \text { implies } \frac{v(A)}{v(B)} \geq \frac{v\left(A^{\prime}\right)}{v\left(B^{\prime}\right)} .
$$

When $A^{\prime}=B^{\prime}, \sum_{a \in A} u(a) \geq \sum_{b \in B} u(b)$ implies $v(A) \geq v(B)$. Therefore, there is an increasing function $f_{i}: \mathbb{R}_{++} \rightarrow \mathbb{R}_{++}$such that $v(A)=f_{i}\left(\sum_{a \in A} u(a)\right)$ for any $A \subseteq X_{i}$. Let $R_{i}=\{x \in$ $\mathbb{R}_{++} \mid x=\sum_{a \in A} u(a)$ for some $\left.A \in \mathscr{A}\right\}$. Then we have for any $v, v^{\prime}, w, w^{\prime} \in R_{i}$,

$$
\frac{v}{v^{\prime}} \geq \frac{w}{w^{\prime}} \text { implies } \frac{f_{i}(v)}{f_{i}\left(v^{\prime}\right)} \geq \frac{f_{i}(w)}{f_{i}\left(w^{\prime}\right)}
$$

Take any $a \in X_{i}$. By Richness, for any $\rho \in(0,1)$, there is $b \in X_{i}$ such that $u(b)=\frac{1-\rho}{\rho} u(a)$. Therefore, for any $\alpha>0$, there is $b \in X_{i}$ such that $u(b)=\alpha$. Hence, $u\left(X_{i}\right)=\mathbb{R}_{++}$.

Take any $\alpha, \beta \in \mathbb{R}_{++}$. Since $u\left(X_{i}\right)=\mathbb{R}_{++}$, there are alternatives $a, b, a^{\prime}, b^{\prime} \in X_{i}$ such that $u(a)=\alpha \beta, u(b)=\beta, u\left(a^{\prime}\right)=\alpha$, and $u\left(b^{\prime}\right)=1$. By the above implication of Relative Likelihood Consistency, we have

$$
\frac{f_{i}(\alpha \beta)}{f_{i}(\beta)}=\frac{f_{i}(\alpha)}{f_{i}(1)} \text { for any } \alpha, \beta>0
$$

Let $g(t) \equiv \frac{f_{i}(t)}{f_{i}(1)}$. Then $g(1)=1$ and $g(\alpha \beta)=g(\alpha) g(\beta)$ for any $\alpha, \beta>0$. Finally, we can prove that $g$ is a power function. Since $g>0$, let $h(t)=\log (g(\exp (t)))$ for any $t \in \mathbb{R}$. Then for any $t, t^{\prime} \in \mathbb{R}$, we have $h\left(t+t^{\prime}\right)=\log \left(g\left(\exp \left(t+t^{\prime}\right)\right)\right)=\log \left(g\left(\exp (t) \exp \left(t^{\prime}\right)\right)\right)=\log \left(g(\exp (t)) g\left(\exp \left(t^{\prime}\right)\right)\right)=$ $\log (g(\exp (t)))+\log \left(g\left(\exp \left(t^{\prime}\right)\right)\right)=h(t)+h\left(t^{\prime}\right)$. We have obtained a typical Cauchy functional equation for $h$. Hence, there is $\eta_{i} \geq 0$ such that $h(t)=\eta_{i} t$. In other words, $g(a)=a^{\eta_{i}}$. Therefore,

$$
\begin{aligned}
p(a, A) & =\frac{f_{i}(1)\left(\sum_{x \in A \cap X_{i}} u(x)\right)^{\eta_{i}}}{\sum_{j: A \cap X_{j} \neq \emptyset} f_{j}(1)\left(\sum_{y \in A \cap X_{j}} u(y)\right)^{\eta_{j}}} \frac{u(a)}{\sum_{b \in A \cap X_{i}} u(b)} . \\
= & \frac{\left(\sum_{x \in A \cap X_{i}} \bar{u}(x)\right)^{\eta_{i}}}{\sum_{j: A \cap X_{j} \neq \emptyset}\left(\sum_{y \in A \cap X_{j}} \bar{u}(y)\right)^{\eta_{j}}} \frac{\bar{u}(a)}{\sum_{b \in A \cap X_{i}} \bar{u}(b)},
\end{aligned}
$$

where $\bar{u}(x)=\left(f_{i}(1)\right)^{\frac{1}{\eta_{i}}} u(x)$ for each $x \in X_{i}$. That is, $p$ is a nested logit.

\section{A.6 Proof of Proposition 3}

Suppose $p$ is the nested logit with $\left(\eta_{1}, \ldots, \eta_{K}, u,\left\{X_{i}\right\}_{i=1}^{K}\right)$ and satisfies Regularity and Richness. If $K=1$, we obtain the desired result since the Luce model is a random utility nested logit. Suppose $K \geq 2$. Let us fix $i \leq K$. We shall prove that $\eta_{i} \leq 1$. Take any $a, a^{\prime} \in X_{i}$ and $b^{\prime} \in X_{j}$ with $i \neq j$. By Richness, for any $\rho \in(0,1)$ there is $b \in X_{j}$ such that $u(b)=\frac{1-\rho}{\rho} u\left(b^{\prime}\right)$. By Regularity, $p(a,\{a, b\}) \leq p\left(a,\left\{a, b, a^{\prime}\right\}\right)$; i.e.,

$$
p(a,\{a, b\})=\frac{(u(a))^{\eta_{i}}}{(u(a))^{\eta_{i}}+(u(b))^{\eta_{j}}} \geq p\left(a,\left\{a, b, a^{\prime}\right\}\right)=\frac{u(a)}{u(a)+u\left(a^{\prime}\right)} \cdot \frac{\left(u(a)+u\left(a^{\prime}\right)\right)^{\eta_{i}}}{\left(u(a)+u\left(a^{\prime}\right)\right)^{\eta_{i}}+(u(b))^{\eta_{j}}} .
$$


After simplifying the above inequality, we obtain

$$
\frac{\left(u(a)+u\left(a^{\prime}\right)\right)^{\eta_{i}}+(u(b))^{\eta_{j}}}{(u(a))^{\eta_{i}}+(u(b))^{\eta_{j}}}=1+\frac{\left(u(a)+u\left(a^{\prime}\right)\right)^{\eta_{i}}-(u(a))^{\eta_{i}}}{(u(a))^{\eta_{i}}+(u(b))^{\eta_{j}}} \geq \frac{\left(u(a)+u\left(a^{\prime}\right)\right)^{\eta_{i}-1}}{(u(a))^{\eta_{i}-1}}
$$

equivalently,

$$
\frac{(u(a))^{\eta_{i}-1}}{(u(a))^{\eta_{i}}+(u(b))^{\eta_{j}}} \geq \frac{\left(u(a)+u\left(a^{\prime}\right)\right)^{\eta_{i}-1}-(u(a))^{\eta_{i}-1}}{\left(u(a)+u\left(a^{\prime}\right)\right)^{\eta_{i}}-(u(a))^{\eta_{i}}} .
$$

Notice that when $\rho$ is close to 0 , we can obtain arbitrary large $u(b)$. Then the left-hand side of above inequality can be arbitrary close to zero. Therefore, the right-hand side must be negative. Hence, since $\eta_{i}>0$ implies $\left(u(a)+u\left(a^{\prime}\right)\right)^{\eta_{i}}-(u(a))^{\eta_{i}}>0$, we have $\eta_{i} \leq 1$.

\section{A.7 Proof of Theorem 4}

Before we proceed to the proof of Theorem 4, it is useful to consider the following generalization of unrestricted cross-nested logit:

$$
p(x, A)=\sum_{k: x \in A \cap X_{k}} \frac{u_{x}^{k}}{\sum_{y \in A \cap X_{k}} u_{y}^{k}} \cdot \frac{\left(\sum_{y \in A \cap X_{k}} u_{y}^{k}\right)^{\lambda}}{\sum_{l: A \cap X_{l} \neq \emptyset}\left(\sum_{z \in A \cap X_{l}} u_{z}^{l}\right)^{\lambda}} .
$$

Note that the representation (14) reduces to unrestricted cross-nested logit by setting $u_{x}^{k}=$ $\left(\alpha_{x}^{k} u(x)\right)^{\frac{1}{\lambda}}$. It turns out that the representation (14) is behaviorally equivalent to the unrestricted cross-nested logit.

Lemma 1. Any stochastic choice p that admits the representation (14) is an unrestricted crossnested logit.

Proof. Suppose that $p$ admits the representation (14) with $\left\{X_{k}\right\}_{k=1}^{K},\left\{u_{x}^{k}\right\}_{k \leq K, x \in X}$, and $\lambda$. Let us define $u: X \rightarrow \mathbb{R}_{++}$and $\alpha_{x}^{k}$ as follows: for each $x \in X$ and $k \leq K$,

$$
u(x) \equiv \sum_{l=1}^{K}\left(u_{x}^{l}\right)^{\lambda} \text { and } \alpha_{x}^{k} \equiv \frac{\left(u_{x}^{k}\right)^{\lambda}}{\sum_{l=1}^{K}\left(u_{x}^{l}\right)^{\lambda}} .
$$

Then we have $u_{x}^{k}=\left(\alpha_{x}^{k} u(x)\right)^{\frac{1}{\lambda}}$ and $\sum_{k=1}^{K} \alpha_{x}^{k}=1$. Hence, we obtain an unrestricted cross-nested logit.

By Lemma 1, we shall prove that any stochastic choice function $p$ admits the representation (14) with some $\left\{X_{k}\right\}_{k=1}^{K},\left\{u_{x}^{k}\right\}_{k \leq K, x \in X}$, and $\lambda$. We first set the collection of subsets $X_{1}, \ldots, X_{K}$ to be equivalent to $\mathscr{A}$. That is, for any $A \in \mathscr{A}$, there $i \leq K$ such that $A=X_{k}$. Moreover, $X_{i} \neq X_{j}$ whenever $i \neq j$. Let us now write $u_{x}^{A}$ rather than $u_{x}^{k}$ when $A=X_{k}$. Then we shall find 
$U=\left\{u_{x}^{A}\right\}_{A \in \mathscr{A}, x \in A} \in \mathbb{R}_{++}^{N}$ where $N \equiv \sum_{A \in \mathscr{A}}|A|$ and $\lambda>0$ such that

$$
p(x, A)=\sum_{B: x \in B} \frac{u_{x}^{B}}{\sum_{y \in A \cap B} u_{y}^{B}} \cdot \frac{\left(\sum_{y \in A \cap B} u_{y}^{B}\right)^{\lambda}}{\sum_{C: A \cap C \neq \emptyset}\left(\sum_{z \in A \cap C} u_{z}^{C}\right)^{\lambda}} .
$$

We prove the above by two steps.

Step 1. There is some function $\sigma: \mathbb{R}_{++}^{N} \rightarrow \mathbb{R}^{N}$ such that $U \in \mathbb{R}_{++}^{N}$ is a fixed point of $\sigma$ iff $p$ admits the representation (15) with respect to $U$.

Let $\tilde{U}=\left\{\tilde{u}_{x}^{A}\right\}_{A \in \mathscr{A}, x \in A}$. Let us define the following mapping for each $U, \tilde{U}$, and $\lambda$ :

$$
\begin{aligned}
q(x, A \mid U, \tilde{U}, \lambda) & \equiv \frac{u_{x}^{A}}{\sum_{y \in A} u_{y}^{A}} \cdot \frac{\left(\sum_{y \in A} \tilde{u}_{y}^{A}\right)^{\lambda}}{\sum_{C: A \cap C \neq \emptyset}\left(\sum_{z \in A \cap C} \tilde{u}_{z}^{C}\right)^{\lambda}} \\
& +\sum_{B: x \in B, A \neq B} \frac{\left(\sum_{y \in A \cap B} \tilde{u}_{y}^{B}\right)^{\lambda}}{\sum_{y \in A \cap B} \tilde{u}_{y}^{B}} \cdot \frac{\left(\sum_{C: A \cap C \neq \emptyset}\left(\sum_{z \in A \cap C} \tilde{u}_{z}^{C}\right)^{\lambda}\right.}{\sum_{C}}
\end{aligned}
$$

Now note that it is enough for us to find $U$ and $\lambda$ such that $p(x, A)=q(x, A \mid U, U, \lambda)$. For notational simplicity, let

$$
f^{A}(\tilde{U}, \lambda) \equiv \frac{\left(\sum_{y \in A} \tilde{u}_{y}^{A}\right)^{\lambda}}{\sum_{C: A \cap C \neq \emptyset}\left(\sum_{z \in A \cap C} \tilde{u}_{z}^{C}\right)^{\lambda}}
$$

and

$$
g_{x}^{A}(\tilde{U}, \lambda) \equiv \sum_{B: x \in B, A \neq B} \frac{\tilde{u}_{x}^{B}}{\sum_{y \in A \cap B} \tilde{u}_{y}^{B}} \cdot \frac{\left(\sum_{y \in A \cap B} \tilde{u}_{y}^{B}\right)^{\lambda}}{\sum_{C: A \cap C \neq \emptyset}\left(\sum_{z \in A \cap C} \tilde{u}_{z}^{C}\right)^{\lambda}} .
$$

Then we have $q(x, A \mid U, \tilde{U}, \lambda)=\frac{u_{x}^{A}}{\sum_{y \in A} u_{y}^{A}} \cdot f^{A}(\tilde{U}, \lambda)+g_{x}^{A}(\tilde{U}, \lambda)$. For any $M \equiv\left\{m^{A}\right\}_{A \in \mathscr{A}} \in \mathbb{R}_{++}^{|\mathscr{A}|}$, let

$$
\sigma_{x}^{A}(\tilde{U}, \lambda, M) \equiv m^{A} \frac{p(x, A)-g_{x}^{A}(\tilde{U}, \lambda)}{f^{A}(\tilde{U}, \lambda)} \text { and } \sigma(\tilde{U}, \lambda, M) \equiv\left\{\sigma_{x}^{A}(\tilde{U}, \lambda, M)\right\}_{A \in \mathscr{A}, x \in A}
$$

Note that $f^{A}(\cdot, \lambda, M)$ and $g_{x}^{A}(\cdot, \lambda, M)$ are strictly positive and continuous functions on $\mathbb{R}_{++}^{N}$. Hence, $\sigma(\cdot, \lambda, M): \mathbb{R}_{++}^{N} \rightarrow \mathbb{R}^{N}$ is continuous for each $(\lambda, M)$. Moreover, 


$$
\begin{aligned}
q(x, A \mid \sigma(\tilde{U}, \lambda, M), \tilde{U}, \lambda) & =\frac{\sigma_{x}^{A}(\tilde{U}, \lambda, M)}{\sum_{y \in A} \sigma_{y}^{A}(\tilde{U}, \lambda, M)} \cdot f^{A}(\tilde{U}, \lambda)+g_{x}^{A}(\tilde{U}, \lambda) \\
& =\frac{m^{A} \frac{p(x, A)-g_{x}^{A}(\tilde{U}, \lambda)}{f^{A}(\tilde{U}, \lambda)}}{\sum_{y \in A} m^{A} \frac{p(y, A)-g_{y}^{A}(\tilde{U}, \lambda)}{f^{A}(\tilde{U}, \lambda)}} \cdot f^{A}(\tilde{U}, \lambda)+g_{x}^{A}(\tilde{U}, \lambda) \\
& =p(x, A) \operatorname{since} f^{A}(\tilde{U}, \lambda)=1-\sum_{x \in A} g_{x}^{A}(\tilde{U}, \lambda) .
\end{aligned}
$$

Therefore, it is enough to find $U \in \mathbb{R}_{++}^{N}$ such that $\sigma(U, \lambda, M)=U$; i.e., a fixed point of $\sigma(\cdot, \lambda, M)$ in $\mathbb{R}_{++}^{N}$. To apply Brouwer's fixed point theorem, ${ }^{22}$ we shall show that for some $(\lambda, M)$ there is a non-empty, convex, compact set $S \subset R_{++}^{N}$ such that $\sigma(\cdot, \lambda, M)$ is a self-map on $S$; that is, $\sigma(\cdot, \lambda, M): S \rightarrow S$.

Step 2. For some $\lambda$ and $M$, there is a non-empty, closed, convex set $S \subset R_{++}^{N}$ such that $\sigma(U, \lambda, M) \in S$ for any $U \in S$. Let $p^{*} \equiv \min \left\{\min _{B \in \mathscr{A}, y \in B} p(y, B), \frac{1}{|X|}\right\}>0$ and

$$
S \equiv\left\{U \in \mathbb{R}_{++}^{N} \mid \sum_{x \in A} u_{x}^{A}=1+\frac{|A|}{|X|^{2}} p^{*} \text { and } \sum_{x \in B} u_{x}^{A} \leq 1-\frac{\left(p^{*}\right)^{2}}{4} \text { for any } A, B \text { with } B \subset A\right\} .
$$

Step 2.1. $S$ is non-empty.

We will show that $U \in S$ when $u_{x}^{A}=\frac{1}{|A|}+\frac{p^{*}}{|X|^{2}}$ for each $A \in \mathscr{A}$ and $x \in X$. First, $\sum_{x \in A} u_{x}^{A}=$ $1+\frac{|A|}{|X|^{2}} p^{*}$. Second, for any $B \subset A$,

$$
\begin{gathered}
\sum_{x \in B} u_{x}^{A} \leq(|A|-1)\left(\frac{1}{|A|}+\frac{p^{*}}{|X|^{2}}\right)=1-\left(\frac{1}{|A|}+\frac{p^{*}}{|X|^{2}}-\frac{|A|}{|X|^{2}} p^{*}\right)<1-\left(\frac{1}{|A|}-\frac{|A|}{|X|^{2}} p^{*}\right) \\
\leq 1-\left(\frac{1}{|X|}-\frac{|X|}{|X|^{2}} p^{*}\right)=1-\frac{\left(1-p^{*}\right)}{|X|} \leq 1-\frac{1}{2|X|} \leq 1-\frac{p^{*}}{2} \leq 1-\frac{\left(p^{*}\right)^{2}}{4} .
\end{gathered}
$$

Step 2.2. $S$ is convex.

Let

$$
S_{1} \equiv\left\{U \in \mathbb{R}_{++}^{N} \mid \sum_{x \in A} u_{x}^{A}=1+\frac{|A|}{|X|^{2}} p^{*}\right\}
$$

and

$$
S_{2} \equiv\left\{U \in \mathbb{R}_{++}^{N} \mid \sum_{x \in B} u_{x}^{A} \leq 1-\frac{\left(p^{*}\right)^{2}}{4} \text { for any } A, B \text { with } B \subset A\right\} .
$$

Note that $S_{1}, S_{2}$ are convex sets. Hence, $S=S_{1} \cap S_{2}$ is convex.

Step 2.3. $S$ is compact.

\footnotetext{
${ }^{22}$ Brouwer's fixed point theorem: Let $S \subset \mathbb{R}^{m}$ be convex and compact and let $f: S \rightarrow S$ be continuous. Then $f$ has a fixed point; that is, there is $s \in S$ such that $f(s)=s$. For example, see Ok (2007), p.279.
} 
Note that $S \subset[0,2]^{N}$. Hence, $S$ is bounded. Moreover, note that $S_{1}, S_{2}$ are closed sets. Hence, $S=S_{1} \cap S_{2}$ is closed.

Step 2.4. For some $\lambda, f^{A}(U, \lambda) \in\left(1-\frac{p^{*}}{2}, 1\right)$ for any $U \in S$.

First, it is immediate that

$$
f^{A}(U, \lambda)=\frac{\left(\sum_{y \in A} u_{y}^{A}\right)^{\lambda}}{\sum_{C: A \cap C \neq \emptyset}\left(\sum_{z \in A \cap C} u_{z}^{C}\right)^{\lambda}}<1 \text { when } U \gg 0 .
$$

Second, in order to show that

$$
f^{A}(U, \lambda)=\frac{\left(\sum_{y \in A} u_{y}^{A}\right)^{\lambda}}{\sum_{C: A \cap C \neq \emptyset}\left(\sum_{z \in A \cap C} u_{z}^{C}\right)^{\lambda}}>1-\frac{p^{*}}{2}
$$

it is enough to prove that

$$
\frac{1}{f^{A}(U, \lambda)}-1=\frac{\sum_{C: A \cap C \neq \emptyset, C \neq A}\left(\sum_{z \in A \cap C} u_{z}^{C}\right)^{\lambda}}{\left(\sum_{y \in A} u_{y}^{A}\right)^{\lambda}}<\frac{p^{*}}{2}
$$

By the construction of $S$, we have $\left(\sum_{y \in A} u_{y}^{A}\right)^{\lambda}=\left(1+\frac{|A|}{|X|^{2}} p^{*}\right)^{\lambda}$. Moreover,

$$
\sum_{C: A \cap C \neq \emptyset, C \neq A}\left(\sum_{z \in A \cap C} u_{z}^{C}\right)^{\lambda}=\sum_{B \subset A}\left(\sum_{y \in B} u_{y}^{B}\right)^{\lambda}+\sum_{C: A \cap C \neq \emptyset, C \nsubseteq A}\left(\sum_{z \in A \cap C} u_{z}^{C}\right)^{\lambda}
$$

and by the construction of $S$,

$$
\sum_{B \subset A}\left(\sum_{y \in B} u_{y}^{B}\right)^{\lambda}=\sum_{B \subset A}\left(1+\frac{|B|}{|X|^{2}} p^{*}\right)^{\lambda}<2^{|X|}\left(1+\frac{|A|-1}{|X|^{2}} p^{*}\right)^{\lambda} .
$$

Moreover, since $C \cap A \neq C$ whenever $C \nsubseteq A$, by the construction of $S$,

$$
\sum_{C: A \cap C \neq \emptyset, C \nsubseteq A}\left(\sum_{z \in A \cap C} u_{z}^{C}\right)^{\lambda} \leq \sum_{C: A \cap C \neq \emptyset, C \nsubseteq A}\left(1-\frac{\left(p^{*}\right)^{2}}{4}\right)^{\lambda}<2^{|X|} .
$$

Combining the last two inequalities, we have

$$
\frac{1}{f^{A}(U, \lambda)}-1=\frac{\sum_{C: A \cap C \neq \emptyset, C \neq A}\left(\sum_{z \in A \cap C} u_{z}^{C}\right)^{\lambda}}{\left(\sum_{y \in A} u_{y}^{A}\right)^{\lambda}}<\frac{2^{|X|}\left(1+\frac{|A|-1}{|X|^{2}} p^{*}\right)^{\lambda}+2^{|X|}}{\left(1+\frac{|A|}{|X|^{2}} p^{*}\right)^{\lambda}} .
$$


Let $\lambda^{*} \equiv \max _{A \in \mathscr{A}} \frac{\log \left(\frac{2|X|+2}{p^{*}}\right)}{\log \left(\frac{1+\frac{|A|}{|X|^{2}} p^{*}}{1+\frac{|A|-1}{|X|^{2}} p^{*}}\right)}$ and $\lambda>\lambda^{*}$. Then we have $\frac{2^{|X|+2}}{p^{*}}<\left(\frac{1+\frac{|A|}{|X|{ }^{2}} p^{*}}{1+\frac{|A|-1}{|X|^{2}} p^{*}}\right)^{\lambda}$. Consequently,

$$
\frac{p^{*}}{2}>\frac{2^{|X|+1}\left(1+\frac{|A|-1}{|X|^{2}} p^{*}\right)^{\lambda}}{\left(1+\frac{|A|}{|X|^{2}} p^{*}\right)^{\lambda}}>\frac{2^{|X|}\left(1+\frac{|A|-1}{|X|^{2}} p^{*}\right)^{\lambda}+2^{|X|}}{\left(1+\frac{|A|}{|X|^{2}} p^{*}\right)^{\lambda}}>\frac{1}{f^{A}(U, \lambda)}-1
$$

Step 2.5. When $\lambda>\lambda^{*}, g_{x}^{A}(U, \lambda) \in\left[0, \frac{p^{*}}{2}\right)$ for any $U \in S$.

It is immediate that $g_{x}^{A}(U, \lambda) \geq 0$. Moreover, since $\sum_{x \in A} g_{x}^{A}(U, \lambda)=1-f^{A}(U, \lambda) \in\left(0, \frac{p^{*}}{2}\right)$ by Step 2.4, $g_{x}^{A}(U, \lambda)<\frac{p^{*}}{2}$.

Step 2.6. Let $m^{A}=1+\frac{|A|}{|X|^{2}} p^{*}$ and $\lambda>\lambda^{*}$. Then $\sigma(U, \lambda, M) \in S$ for any $U \in S$.

To show that $\sigma(U, \lambda, M) \in S$, we shall prove that for any $A \in \mathscr{A}$, (i) $\sigma_{x}^{A}(U, \lambda, M)>0$, (ii) $\sum_{x \in A} \sigma_{x}^{A}(U, \lambda, M)=1+\frac{|A|}{|X|^{2}} p^{*}$, and (iii) $\sum_{y \in B} \sigma_{y}^{A}(U, \lambda, M) \leq 1-\frac{\left(p^{*}\right)^{2}}{4}$ for any $B \subset A$.

Step 2.6.(i). $\sigma_{x}^{A}(U, \lambda, M)>\frac{m^{A} p^{*}}{2}$.

By Step 2.5 and the definition of $p^{*}$, we have $p(x, A) \geq p^{*}$ and $\frac{p^{*}}{2}>g_{x}^{A}(U, \lambda)$. Therefore, since $f^{A}(U, \lambda)<1, \sigma_{x}^{A}(U, \lambda, M)=m^{A} \frac{p(x, A)-g_{x}^{A}(U, \lambda)}{f^{A}(U, \lambda)}>m^{A} \frac{\frac{p^{*}}{2}}{f^{A}(U, \lambda)}>\frac{m^{A} p^{*}}{2}$.

Step 2.6.(ii). $\sum_{x \in A} \sigma_{x}^{A}(U, \lambda, M)=1+\frac{|A|}{|X|^{2}} p^{*}$.

Since $f^{A}(U, \lambda)=1-\sum_{x \in A} g_{x}^{A}(U, \lambda)$ and by the definition of $m^{A}$, $\sum_{x \in A} \sigma_{x}^{A}(U, \lambda, M)=\sum_{x \in A} m^{A} \frac{p(x, A)-g_{x}^{A}(U, \lambda)}{f^{A}(U, \lambda)}=m^{A} \frac{1-\sum_{x \in A} g_{x}^{A}(U, \lambda)}{f^{A}(U, \lambda)}=m^{A}=1+\frac{|A|}{|X|^{2}} p^{*}$.

Step 2.6.(iii). $\sum_{y \in B} \sigma_{y}^{A}(U, \lambda, M) \leq 1-\frac{\left(p^{*}\right)^{2}}{4}$ for any $B \subset A$.

Suppose $x \in A \backslash B$. Then by Step 2.6.(i),

$$
\sum_{y \in B} \sigma_{y}^{A}(U, \lambda, M) \leq \sum_{y \in A} \sigma_{y}^{A}(U, \lambda, M)-\sigma_{x}^{A}(U, \lambda, M)=m^{A}-\sigma_{x}^{A}(U, \lambda, M)<m^{A}-\frac{m^{A} p^{*}}{2}
$$

Finally,

$$
m^{A}-\frac{m^{A} p^{*}}{2}=\left(1-\frac{p^{*}}{2}\right)\left(1+\frac{|A|}{|X|^{2}} p^{*}\right) \leq\left(1-\frac{p^{*}}{2}\right)\left(1+\frac{|X|}{|X|^{2}} p^{*}\right) \leq\left(1-\frac{p^{*}}{2}\right)\left(1+\frac{1}{2} p^{*}\right)=1-\frac{\left(p^{*}\right)^{2}}{4} .
$$

To sum up, Step 1 shows that $p$ admits the representation (15) with respect to $U$ if and only if $U$ is a fixed point of $\sigma$. Step 2 shows that $\sigma$ has a fixed point by Brouwer's fixed point theorem. Therefore, by Steps 1-2 and Lemma 1, any $p$ is an unrestricted cross-nested logit. 


\section{A.8 Proof of Proposition 4}

We use standard strong consistency results for M-estimators (e.g., see p. 2121-2 of Newey and McFadden (1994)). To obtain $\hat{\mathcal{X}} \stackrel{\text { a.s. }}{\longrightarrow} \mathcal{X}^{*}$, since the set of all nest structures is finite, we only need to prove that $\mathcal{X}^{*}$ is the unique minimizer of $D^{*}$ and $D \stackrel{\text { a.s. }}{\longrightarrow} D^{*}$.

We first simplify the calculation of $D_{1}(\mathcal{Y})$. Let $\bar{\epsilon}_{B, A} \equiv \frac{\sum_{a \in A \cap B} \epsilon_{a, A}}{\bar{p}(A \cap B, A)}$. Then $r_{A}(a, b)=\frac{\bar{p}(a, A)+\epsilon_{a, A}}{\bar{p}(b, A)+\epsilon_{b, A}}=$ $\bar{r}_{A}(a, b) \frac{1+\bar{\epsilon}_{a, A}}{1+\bar{\epsilon}_{b, A}}$. Let

$$
\zeta_{a, b, A, B} \equiv \log \left(\frac{1+\bar{\epsilon}_{a, A}}{1+\bar{\epsilon}_{b, A}} / \frac{1+\bar{\epsilon}_{a, B}}{1+\bar{\epsilon}_{b, B}}\right) \text { and } \delta_{a, b, A, B} \equiv \log \left(\frac{\bar{r}_{A}(a, b)}{\bar{r}_{B}(a, b)}\right)
$$

Then

$$
\log \left(\frac{r_{A}(a, b)}{r_{B}(a, b)}\right)=\delta_{a, b, A, B}+\zeta_{a, b, A, B}
$$

We now simplify the calculation of $D_{2}(\mathcal{Y})$. Note that

$$
r_{A}\left(Y, Y^{\prime}\right)=\frac{p(A \cap Y, A)}{p\left(A \cap Y^{\prime}, A\right)}=\frac{\bar{p}(A \cap Y, A)+\sum_{a \in A \cap Y} \epsilon_{a, A}}{\bar{p}\left(A \cap Y^{\prime}, A\right)+\sum_{a \in A \cap Y^{\prime}} \epsilon_{a, A}}=\bar{r}_{A}\left(Y, Y^{\prime}\right) \frac{1+\bar{\epsilon}_{Y, A}}{1+\bar{\epsilon}_{Y^{\prime}, A}} .
$$

Similarly, let

$$
\zeta_{Y, Y^{\prime}, A, B} \equiv \log \left(\frac{1+\bar{\epsilon}_{Y, A}}{1+\bar{\epsilon}_{Y^{\prime}, A}} / \frac{1+\bar{\epsilon}_{Y, B}}{1+\bar{\epsilon}_{Y^{\prime}, B}}\right) \text { and } \delta_{Y, Y^{\prime}, A, B} \equiv \log \left(\frac{\bar{r}_{A}\left(Y, Y^{\prime}\right)}{\bar{r}_{B}\left(Y, Y^{\prime}\right)}\right)
$$

Then

$$
\log \left(\frac{r_{A}\left(Y, Y^{\prime}\right)}{r_{B}\left(Y, Y^{\prime}\right)}\right)=\delta_{Y, Y^{\prime}, A, B}+\zeta_{Y, Y^{\prime}, A, B}
$$

Let $N_{1}(\mathcal{Y})=\sum_{Y \in \mathcal{Y}}|\{(A, B, a, b) \mid a, b \in A \cap B \cap Y\}|$ and $N_{2}(\mathcal{Y})=\sum_{Y, Y^{\prime} \in \mathcal{Y}} \mid\{(A, B) \mid A \cap Y=$ $\left.B \cap Y, A \cap Y^{\prime}=B \cap Y^{\prime}\right\} \mid$ and take any $M>\max \mathcal{y} N_{1}(\mathcal{Y}), \max \mathcal{Y} N_{2}(\mathcal{Y})$. Hence,

$$
\begin{gathered}
D(\mathcal{Y})=\frac{\sum_{Y \in \mathcal{Y}} \sum_{A, B \in \mathscr{A}, a, b \in A \cap B \cap Y}\left(\delta_{a, b, A, B}+\zeta_{a, b, A, B}\right)^{2}}{N_{1}(\mathcal{Y})} \\
+\frac{\sum_{Y, Y^{\prime} \in \mathcal{Y}} \sum_{A, B \in \mathscr{A}: A \cap Y=B \cap Y, A \cap Y^{\prime}=B \cap Y^{\prime}}\left(\delta_{Y, Y^{\prime}, A, B}+\zeta_{Y, Y^{\prime}, A, B}\right)^{2}}{N_{2}(\mathcal{Y})} .
\end{gathered}
$$

We then show that $\mathcal{X}^{*}$ is the unique minimizer of $D^{*}$. Since $D^{*}\left(\mathscr{X}^{*}\right)=0$, we shall show that $D^{*}(\mathcal{Y})>0$ for any nest structure $\mathcal{Y} \neq \mathcal{X}^{*}$. It is enough to consider the following two cases.

Case 1. $\mathcal{Y}$ is a partition of $X$ such that there are $a \in X_{i}$ and $b \in X_{j}$ such that $a, b \in Y$ for some $Y \in \mathcal{Y}$.

By Assumption 1, there are $A, B$ with $\bar{r}_{A}(a, b) \neq \bar{r}_{B}(a, b)$; i.e., $\delta_{a, b, A, B} \neq 0$. Then we have $D^{*}(\mathcal{Y}) \geq D_{1}^{*}(\mathcal{Y})>\left(\delta_{a, b, A, B}\right)^{2} / M>0$.

Case 2. $\mathcal{Y}$ is a partition of $X$ such that for any $Y \in \mathcal{Y}, Y \subseteq X_{i}$ for some $i$, and $Y^{\prime} \subset X_{j}$ for some $Y^{\prime} \in \mathcal{Y}$ and $j$. 
Take any $Y, Y^{\prime}$ such that $Y \subset X_{i}$ and $Y^{\prime} \subseteq X_{j}$. By Assumption 1, there are $A, B$ such such that $\bar{r}_{A}\left(Y, Y^{\prime}\right) \neq \bar{r}_{B}\left(Y, Y^{\prime}\right), A \cap Y=B \cap Y$, and $A \cap Y^{\prime}=B \cap Y^{\prime}$. That is, $\delta_{Y, Y^{\prime}, A, B} \neq 0$. Then we have $D^{*}(\mathcal{Y}) \geq D_{2}^{*}(\mathcal{Y}) \geq\left(\delta_{Y, Y^{\prime}, A, B}\right)^{2} / M>0$.

We finally show that $D(\mathcal{Y}) \stackrel{\text { a.s. }}{\longrightarrow} D^{*}(\mathcal{Y})$ for every $\mathcal{Y}$. We have

$$
\begin{aligned}
D(\mathcal{Y})-D^{*}(\mathcal{Y}) & =\sum \frac{\left(\left(\delta_{a, b, A, B}+\zeta_{a, b, A, B}\right)^{2}-\left(\delta_{a, b, A, B}\right)^{2}\right)}{N_{1}(\mathcal{Y})}+\sum \frac{\left(\left(\delta_{Y, Y^{\prime}, A, B}+\zeta_{Y, Y^{\prime}, A, B}\right)^{2}-\left(\delta_{Y, Y^{\prime}, A, B}\right)^{2}\right)}{N_{2}(\mathcal{Y})} \\
& =\sum \frac{\zeta_{a, b, A, B}\left(2 \delta_{a, b, A, B}+\zeta_{a, b, A, B}\right)}{N_{1}(\mathcal{Y})}+\sum \frac{\zeta_{Y, Y^{\prime}, A, B}\left(2 \delta_{Y, Y^{\prime}, A, B}+\zeta_{Y, Y^{\prime}, A, B}\right)}{N_{2}(\mathcal{Y})} \stackrel{a . s .}{\longrightarrow} 0
\end{aligned}
$$

since $\zeta_{a, b, A, B} \stackrel{a . s .}{\longrightarrow} 0, \zeta_{Y, Y^{\prime}, A, B} \stackrel{a . s .}{\longrightarrow} 0$, and $\delta_{a, b, A, B}$ and $\delta_{Y, Y^{\prime}, A, B}$ are constants.

\section{A.9 Proof of Proposition 5}

Take any $a, b \in X$. As we showed in the proof of Proposition 6 , we have $\log \left(r_{A}(a, b) / r_{B}(a, b)\right)=$ $\delta_{a, b, A, B}+\zeta_{a, b, A, B}$. If $a, b \in X_{i}$ for some $i$, then

$$
d(a, b)=\frac{\sum_{A, B \in \mathscr{A}: a, b \in A \cap B} \zeta_{a, b, A, B}^{2}}{|\{(A, B, a, b) \mid a, b \in A \cap B\}|} \stackrel{a . s .}{\longrightarrow} 0 .
$$

If $a \in X_{i}$ and $b \in X_{j}$ for some $i, j$ with $i \neq j$, then by Assumption 1 , there are $A^{*}, B^{*}$ with $\bar{r}_{A^{*}}(a, b) \neq \bar{r}_{B^{*}}(a, b) ;$ i.e., $\delta_{a, b, A^{*}, B^{*}} \neq 0$. Hence,

$$
d(a, b)>\frac{\left(\delta_{a, b, A^{*}, B^{*}}+\zeta_{a, b, A^{*}, B^{*}}\right)^{2}}{M} \geq \frac{\left(\delta_{a, b, A^{*}, B^{*}}\right)^{2}}{2 M} \text { almost surely. }
$$

Let

$$
\epsilon^{*} \equiv \min _{a^{\prime}, b^{\prime}, A^{\prime}, B^{\prime}: \delta_{a^{\prime}, b^{\prime}, A^{\prime}, B^{\prime}} \neq 0} \frac{\left(\delta_{a^{\prime}, b^{\prime}, A^{\prime}, B^{\prime}}\right)^{2}}{2 M} .
$$

Then by the previous inequality, $d(a, b)>\epsilon^{*}$ almost surely. Since $\epsilon^{*}>0$, there is $\bar{N}$ such that for any $N^{*}>\bar{N}$,

$$
\max _{i} \max _{a, b \in X_{i}} d(a, b)<\epsilon^{*}<\min _{i<j} \min _{a^{\prime} \in X_{i}, b^{\prime} \in X_{j}} d\left(a^{\prime}, b^{\prime}\right) \text { with probability one. }
$$

\section{A.10 Proofs of Proposition 6 and Corollary 2}

Proof of Proposition 6. Since $X$ and $\mathscr{A}$ are finite, there is a set $\left\{d_{1}, \ldots, d_{m}\right\}$ of positive real numbers such that $\max _{a, b} d(a, b)=d_{m}>\ldots>d_{2}>d_{1}=\min _{a, b} d(a, b) \geq 0$ and for any $a^{\prime}, b^{\prime} \in X$, $d\left(a^{\prime}, b^{\prime}\right)=d_{s}$ for some $s \leq m$. Hence, it is immediate that $\left|\mathscr{X}^{*}\right| \leq|X|^{2}$.

Let us prove $\left|\mathscr{X}^{*}\right| \leq|X|$ by induction on the number of alternatives $|X|$. When $|X|=2$, we have $\left|\mathscr{X}^{*}\right| \leq|\mathscr{X}|=2=|X|$. Suppose that the hypothesis is true for any set $X$ with $|X|=k$. We shall prove that this also holds for all sets $X$ with $|X|=k+1$.

Take a set $X$ and suppose $|X|=k+1$. Let $a^{*}, b^{*}$ be elements of $X$ such that $d_{1}=d\left(a^{*}, b^{*}\right)$. 
Hence, for any $\epsilon \in\left(d_{1}, d_{m}\right], a^{*} \sim_{\epsilon} b^{*}$. In other words, $a^{*}$ and $b^{*}$ belong to the same nest for any partition $\mathcal{X}_{\epsilon}$ with $\epsilon \in\left(d_{1}, d_{m}\right]$. If $d_{1}=d\left(a^{*}, b^{*}\right)=d_{m}=\max _{a, b \in X} d(a, b)$, then we obtain the desired result since $\left|\mathscr{X}^{*}\right|=1$. Suppose that $d\left(a^{*}, b^{*}\right)<d_{m}$. Then, without loss of generality, we can assume that $d_{m}=\max _{a, b \in X^{\prime}} d(a, b)$ where $X^{\prime}=X \backslash\left\{b^{*}\right\}$. Because $\left|X^{\prime}\right| \leq k$, it follows from the induction assumption that there are at most $k$ different partitions in $\mathscr{X}^{\prime *}=\left\{\mathcal{X}_{\epsilon}^{\prime}\right\}_{\epsilon \in\left[0, d_{m}\right]}$.

Now let us consider $\mathscr{X}^{*}$. When $\epsilon \in\left(d_{1}, d_{m}\right]$, adding $b^{*}$ to $X^{\prime}$ does not increase the number of distinct partitions in $\left\{\mathcal{X}_{\epsilon}^{\prime}\right\}_{\epsilon \in\left[0, d_{m}\right]}$ since $a^{*}$ and $b^{*}$ must belong to the same nest. Note that if $\sim_{\epsilon}$ is transitive on $X$, then it is also transitive on $X^{\prime}$. Hence, adding $b^{*}$ to $X^{\prime}$ does not extend the set of $\epsilon$ such that $\sim_{\epsilon}$ is transitive. Therefore, there is at most one new partition when $b^{*}$ is added to $X^{\prime}$. Therefore, by induction, $\left|\mathscr{X}^{*}\right| \leq|X|$.

Proof of Corollary 2. In the proof of Proposition 6, we show that there is $\epsilon^{*}>0$ such that

$$
\max _{i} \max _{a, b \in X_{i}} d(a, b)<\epsilon^{*}<\min _{i<j} \min _{a^{\prime} \in X_{i}, b^{\prime} \in X_{j}} d\left(a^{\prime}, b^{\prime}\right) \text { almost surely. }
$$

Hence $\mathcal{X}_{\epsilon^{*}}=\mathcal{X}^{*}$ almost surely. Therefore, $\mathcal{X}^{*} \in \mathscr{X}^{*}$ almost surely. By Proposition $6, \hat{\mathcal{X}} \stackrel{\text { a.s. }}{\longrightarrow} \mathcal{X}^{*}$. Therefore, since $\mathcal{X}^{*} \in \mathscr{X}^{*}$ almost surely and $\mathscr{X}^{*} \subset \mathscr{X}$, we have $\hat{\mathcal{X}}^{*} \stackrel{\text { a.s. }}{\longrightarrow} \mathcal{X}^{*}$.

\section{B Additional Results}

\section{B.1 Regularity, Increasing NSC, and The Similarity Effect}

Proposition 3 shows that regularity has important behavioral implications for nested logit. In this section, we study the implications of regularity for general NSC. We show that there is a deep connection between regularity, increasing NSCs, and the similarity effect. To clarify the implications of regularity, we divide regularity into two logically independent axioms (as we did for IIA).

Axiom 6 (Dissimilar Regularity). For any $A \in \mathscr{A}, x \in A$, and $y \in X$,

$$
p(x, A \cup y) \leq p(x, A) \text { when } x \nsim_{p} y .
$$

Axiom 7 (Similar Regularity). For any $A \in \mathscr{A}, x \in A$, and $y \in X$,

$$
p(x, A \cup y) \leq p(x, A) \text { when } x \sim_{p} y .
$$

The first axiom, Dissimilar Regularity, says that regularity should hold when $x$ and $y$ are revealed dissimilar, while the second axiom, Similar Regularity, requires regularity when $x$ and $y$ are revealed similar. It is immediate that the joint assumption of Dissimilar Regularity and Similar Regularity is equivalent to regularity.

The first result shows that Similar Regularity is closely related to the similarity effect. In fact, the similarity effect implies Similar Regularity in a setting that is more general than NSC. 
Proposition 7. For any stochastic choice function $p$, if $\sim_{p}$ is transitive, then the similarity effect implies Similar Regularity.

The intuition behind this result is quite simple. If an alternative $y$ is introduced and it is similar to some existing alternative $x$, the similarity effect requires that $y$ hurts $x$ more than it hurts anything that it is not similar to. Thus, the probability of $x$ must decrease. Note that this result only requires that $\sim_{p}$ is transitive; it does not rely on the structure of NSC. Consequently, it is difficult to explain both the similarity effect and violations of regularity. Further, we can show that under some richness condition, Similar Regularity will imply a weak version of the similarity effect, and thus the similarity effect is essentially equivalent to Similar Regularity.

The second result shows that Dissimilar Regularity is equivalent to a mild but behaviorally important restriction on $v$ : monotonicity in the size of the nest. We say $p$ is an increasing NSC if $v(A) \geq v(B)$ for any $i \leq K$ and nonempty sets $A, B \subseteq X_{i}$ with $B \subseteq A$.

Proposition 8. A nondegenerate NSC p satisfies Dissimilar Regularity if and only if it is an increasing NSC.

Increasing NSC are interesting because they subsume many of the models in the literature, including nested logit. However, increasing NSC are incompatible with certain violations of regularity, such as choice overload. In fact, Propositions 7 and 8 imply that increasing NSC cannot allow the similarity effect and violations of regularity simultaneously. Thus, non-increasing NSC (e.g., the menu-dependent substitutability example from section 3.4) are of independent interest.

\section{B.2 Alternative Axiomatization of NSC}

In this section, we provide an alternative axiomatic characterization of NSC in which characterizing axioms do not rely on our revealed similarity relation $\sim_{p}$. To characterize NSC, we "divide" ISA into two axioms.

Axiom 8 (ISA-1). For any $A \in \mathscr{A}, a, b \in A$, and $x \notin A$,

$$
\frac{p(a,\{a, x\})}{p(x,\{a, x\})}=\frac{p(a,\{a, b, x\})}{p(x,\{a, b, x\})} \text { and } \frac{p(b,\{b, x\})}{p(x,\{b, x\})}=\frac{p(b,\{a, b, x\})}{p(x,\{a, b, x\})} \Longrightarrow \frac{p(a, A)}{p(b, A)}=\frac{p(a, A \cup x)}{p(b, A \cup x)}
$$

Note that the above axiom is essentially identical to the first part of ISA with $a \sim_{p} x$ and $b \sim_{p} x$.

Axiom 9 (ISA-2). For any $A, B, C \in \mathscr{A}, a \in A \cap B, b \in A \cap C$, and $x \in B \cap C$,

$$
\frac{p(a,\{a, x\})}{p(x,\{a, x\})} \neq \frac{p(a, B)}{p(x, B)} \text { and } \frac{p(b,\{b, x\})}{p(x,\{b, x\})} \neq \frac{p(b, C)}{p(x, C)} \Longrightarrow \frac{p(a, A)}{p(b, A)}=\frac{p(a, A \cup x)}{p(b, A \cup x)}
$$

Similarly, the above axiom is essentially identical to the second part of ISA with $a \chi_{p} x$ and $b \chi_{p} x$. 
We also need to strengthen our notion of nondegeneracy as follows. The NSC $p$ with $\left(v, u,\left\{X_{i}\right\}_{i=1}^{N}\right)$ is strict if for any $A_{i} \subset X_{i}$ with $a \in A_{i}$ and $x \in X_{i} \backslash A_{i}$,

$$
\text { if } \frac{u(a)+u(x)}{u(x)}=\frac{v(\{a, x\})}{v(x)} \text {, then } \frac{\sum_{a^{\prime} \in A_{i}} u\left(a^{\prime}\right)+u(x)}{\sum_{a^{\prime} \in A_{i}} u\left(a^{\prime}\right)}=\frac{v\left(A_{i} \cup x\right)}{v\left(A_{i}\right)} \text {. }
$$

Theorem 5. Let $p$ be a stochastic choice function with at least three alternatives that are dissimilar to each other. Then $p$ satisfies AxIOM 8 and Axiom 9 if and only if it is a strict nondegenerate $N S C$.

\section{B.3 Three-Step Nested Stochastic Choice}

Theorem 1 shows that all two-level nested logit models are in fact special cases of NSC and are characterized by a strong notion of categorical similarity. A natural question is, can we capture more complex substitution patterns through a more general notion of similarity? Put another way, can we allow for contextual or comparative similarity?

Consider a decision maker who is choosing between wines and beers. As in NSC, it is natural to think that there are three nests: one for white wines, one for red wines, and one for beers. Intuitively, the wines are "more similar" to each other than they are to the beers. This can be captured though an intermediate step in which, before deciding between red or white wines, the decision maker decides between wines and beers. After deciding between wine and beer, the consumer decides between different styles of wine (red vs. white), and then selects a specific one to consume. This can be represented through a three-level nested structure, which we refer to as a 3-step NSC. In this section, we show that we can capture such complex relationships through the introduction of a second similarity relation and a generalization of our main axiom.

Formally, any 3-step NSC consists of a nesting structure (tree) and conditional Luce rules.

Definition 8 (3-step NSC). A stochastic choice function $p$ is a 3-Step Nested Stochastic Choice if there exist a partition $X_{1}, \ldots, X_{K}$ of $X$, a partition $X_{k}^{1}, \ldots, X_{k}^{q_{k}}$ of $X_{k}$ for each $k \leq K$, and functions $u: X \rightarrow \mathbb{R}_{++}, w: \bigcup_{i=1}^{K} 2^{X_{i}} \rightarrow \mathbb{R}_{+}$, and $v: \bigcup_{k=1}^{K} \bigcup_{l=1}^{q_{k}} 2^{X_{k}^{l}} \rightarrow \mathbb{R}_{+}$with $w(\emptyset)=v(\emptyset)=0$ such that for any $A \in \mathscr{A}$ and $x \in A \cap X_{k}^{j}$,

$$
p(x, A)=\frac{u(x)}{\sum_{y \in A \cap X_{k}^{j}} u(y)} \cdot \frac{v\left(A \cap X_{k}^{j}\right)}{\sum_{l=1}^{q_{k}} v\left(A \cap X_{k}^{l}\right)} \cdot \frac{w\left(A \cap X_{k}\right)}{\sum_{i=1}^{K} w\left(A \cap X_{i}\right)} .
$$

We now introduce a secondary notion of similarity which applies to alternatives that are not categorically similar, but satisfy IIA in the presence of mutually dissimilar alternatives.

Definition 9. For any $a, b \in X$, we say $a$ and $b$ are approximately revealed similar, denoted by $a \bowtie_{p} b$, if $a \nsim_{p} b$ and

$$
\frac{p(a, A)}{p(b, A)}=\frac{p(a, A \cup x)}{p(b, A \cup x)} \text { for any } A \in \mathscr{A} \text { and } x \notin A \text { with } x \not_{p} a \text { and } x \not_{p} b \text {. }
$$


We write $a \simeq_{p} b$ if either $a \sim_{p} b$ and $a \bowtie_{p} b$.

It is crucial to note that our approximately revealed similar relation requires that $a$ and $b$ are not categorically similar. Thus we have two distinct "layers" of similarity; $\bowtie_{p}$ does not include $\sim_{p}$ as a sub-relation. In terms of our drink example, all the red wines are categorically similar (related through $\sim_{p}$ ), while red and white wines are approximately similar (related through $\bowtie_{p}$ ), as IIA will hold between them when a beer is introduced but not if another wine were introduced. Hence this second layer delineates the "intermediate" nests in the tree and captures aspects of context-dependent similarity. Consequently, this approach distinguishes between fundamental and contextual similarity.

We now introduce a generalization of our main axiom, to characterize 3-step NSC.

Axiom 10 (Generalized Independence of Symmetric Alternatives). For any $A \in \mathscr{A}, a, b \in A$, and $x \notin A$,

$$
\begin{aligned}
& a \sim_{p} x \text { and } b \sim_{p} x, \\
& a \bowtie_{p} x \text { and } b \bowtie_{p} x, \\
& \quad \text { or } \\
& a \neq_{p} x \text { and } b \neq_{p} x
\end{aligned}
$$

Recall that the second part of Independence of Symmetric Alternatives requires that $\frac{p(a, A)}{p(b, A)}=$ $\frac{p(a, A \cup x)}{p(b, A \cup x)}$ when $a \chi_{p} x$ and $b \nsim_{p} x$. However, the second part of Generalized Independence of Symmetric Alternatives requires that $\frac{p(a, A)}{p(b, A)}=\frac{p(a, A \cup x)}{p(b, A \cup x)}$ when either $a \bowtie_{p} x$ and $b \bowtie_{p} x$ or $a \varkappa_{p} x$ and $b \varkappa_{p} x$. Hence, Generalized Independence of Symmetric Alternatives relaxes the second part of Independence of Symmetric Alternatives.

Lastly, we need a consistency condition to hold between the similarity relations.

Axiom 11 (Consistency of Revealed Similarities). For any $x, y, x^{\prime} \in X$, if $x \sim_{p} x^{\prime}$, then

$$
x \bowtie_{p} y \text { if and only if } x^{\prime} \bowtie_{p} y .
$$

In the language of our drink example, $y$ is a white wine and $x, x^{\prime}$ are two red wines. Since $x \sim_{p} x^{\prime}$, it must be the case that if a white wine $y$ is approximately similar to some red wine $x$, then it is approximately similar to any other red wine $x^{\prime}$.

Theorem 6. Consider a stochastic choice function $p$ such that there are $a, b, c$ with $a \neq_{p} b, b \neq_{p} c$, and $a \Varangle_{p} c$. Suppose, for any $x \in X$, there are $y, z \in X$ such that $x \bowtie_{p} y, y \bowtie_{p} z$, and $x \bowtie_{p} z$. If $p$ satisfies Generalized Independence of Symmetric Alternatives and Consistency of Revealed Similarities, then it is a 3-step NSC. ${ }^{23}$

\footnotetext{
${ }^{23}$ Indeed, just as in Theorem 1 , the necessity direction also holds when the appropriate nondegeneracy condition is imposed on $p$.
} 
The major insight from this result is that multi-step NSC is characterized by revealing multiple, layered similarity relations, and then imposing a generalization of our key axiom. Just as our similarity relation identifies endogenous nests, this secondary relation identifies endogenous, intermediate nests. Thus, Theorem 6 shows that we may identify an endogenous tree structure.

Multi-level nested logit models have been applied to many situations. Most famously, Goldberg (1995) uses a multi-level nested logit to study automobile demand. It is well known that the "order" in which the tree-structure of nests is specified matters for estimates. Our approach reveals the entire, endogenous tree, and so the "order" is also recovered: $\bowtie_{p}$ captures upper nests and $\sim_{p}$ captures lower nests. It is straightforward to see how our approach could be extended to characterize an $N$-Step NSC.

\section{B.4 Remaining Proofs}

\section{B.4.1 Proof of Proposition 7}

As discussed in the proof of Theorem 1 , when $\sim_{p}$ is transitive, there is a partition $\left\{X_{i}\right\}_{i=1}^{K}$ such that for any $x, y \in X, x \sim_{p} y$ if and only if $x, y \in X_{i}$ for some $i \leq K$. Suppose that $p$ satisfies the similarity effect; that is, for any $A \in \mathscr{A}$ and $a, a^{\prime} \in X_{i}$ and $b \in X_{j}$ with $a, b \in A$ and $a^{\prime} \notin A$,

$$
\frac{p(a, A)}{p(b, A)}>\frac{p\left(a, A \cup a^{\prime}\right)}{p\left(b, A \cup a^{\prime}\right)} .
$$

Step 1. For any $A \in \mathscr{A}$ and $a^{\prime} \in X_{i} \backslash A$ with $A \cap X_{i} \neq \emptyset, p\left(A \cap X_{i}, A\right)>p\left(A \cap X_{i}, A \cup a^{\prime}\right)$.

The similarity effect implies that for any $b \in A \backslash X_{i}$ and $a \in A \cap X_{i}$,

$$
p\left(b, A \cup a^{\prime}\right) p(a, A)>p(b, A) p\left(a, A \cup a^{\prime}\right) .
$$

Let us first add Inequality (16) across all $a \in A \cap X_{i}$. Then we have

$$
p\left(b, A \cup a^{\prime}\right) p\left(A \cap X_{i}, A\right)>p(b, A) p\left(A \cap X_{i}, A \cup a^{\prime}\right) .
$$

Let us add again the above inequality across all $b \in A \backslash X_{i}$. Then we obtain

$$
\left(1-p\left(A \cap X_{i}, A \cup a^{\prime}\right)-p\left(a^{\prime}, A \cup a^{\prime}\right)\right) p\left(A \cap X_{i}, A\right)>\left(1-p\left(A \cap X_{i}, A\right)\right) p\left(A \cap X_{i}, A \cup a^{\prime}\right) .
$$

The above inequality implies

$$
\left(1-p\left(A \cap X_{i}, A \cup a^{\prime}\right)\right) p\left(A \cap X_{i}, A\right)>\left(1-p\left(A \cap X_{i}, A\right)\right) p\left(A \cap X_{i}, A \cup a^{\prime}\right) ;
$$

equivalently, $p\left(A \cap X_{i}, A\right)>p\left(A \cap X_{i}, A \cup a^{\prime}\right)$.

Step 2. For any $A \in \mathscr{A}, a \in A \cap X_{i}$, and $a^{\prime} \in X_{i} \backslash A, p(a, A)>p\left(a, A \cup a^{\prime}\right)$.

For any $\tilde{a} \in A \cap X_{i}$, we have $\frac{p\left(a, A \cup a^{\prime}\right)}{p\left(\tilde{a}, A \cup a^{\prime}\right)}=\frac{p(a, A)}{p(\tilde{a}, A)}$. Let us add the equality $p\left(a, A \cup a^{\prime}\right) p(\tilde{a}, A)=$ 
$p(a, A) p\left(\tilde{a}, A \cup a^{\prime}\right)$ for all $\tilde{a} \in A \cap X_{i}$. Then we obtain $p\left(a, A \cup a^{\prime}\right) p\left(A \cap X_{i}, A\right)=p(a, A) p(A \cap$ $\left.X_{i}, A \cup a^{\prime}\right)$. Finally, since $p\left(A \cap X_{i}, A \cup a^{\prime}\right)<p\left(A \cap X_{i}, A\right)$, we need to have $p(a, A)>p\left(a, A \cup a^{\prime}\right)$.

\section{B.4.2 Proof of Proposition 8}

Let $p$ be a nondegenerate NSC with $\left(v, u,\left\{X_{i}\right\}_{i=1}^{K}\right)$.

Sufficiency. Take any $A_{j} \subset X_{j}$ and $y \in X_{j} \backslash A_{j}$. Take any $x \in X_{i}$ with $i \neq j$. Since $x \neq_{p} y$, by Dissimilar Regularity, we have

$$
p\left(x, A_{j} \cup x \cup y\right)=\frac{v(x)}{v(x)+v\left(A_{j} \cup y\right)} \leq p\left(x, A_{j} \cup x\right)=\frac{v(x)}{v(x)+v\left(A_{j}\right)} \text { iff } v\left(A_{j}\right) \leq v\left(A_{j} \cup y\right) .
$$

Necessity. Suppose $v$ is increasing. Take any $A \in \mathscr{A}, x \in A$, and $y \notin A$ with $x \varkappa_{p} y$. Therefore, $x \in X_{i}$ and $y \in X_{j}$ for some $i, j$ with $i \neq j$. Since $v\left(A \cap X_{j} \cup y\right) \geq v\left(A \cap X_{j}\right)$, we have

$$
p(x, A \cup y)=\frac{p\left(x, A \cap X_{i}\right) v\left(A \cap X_{i}\right)}{v\left(A_{j} \cup y\right)+\sum_{k \neq j} v\left(A \cap X_{k}\right)} \leq p(x, A)=\frac{p\left(x, A \cap X_{i}\right) v\left(A \cap X_{i}\right)}{v\left(A_{j}\right)+\sum_{k \neq j} v\left(A \cap X_{k}\right)} .
$$

\section{B.4.3 Proof of Theorem 5}

Sufficiency. Take any $A \in \mathscr{A}, a, b \in A$, and $x \notin A$. Suppose $a \sim_{p} x$ and $b \sim_{p} x$. Then we have $\frac{p(a,\{a, x\})}{p(x,\{a, x\})}=\frac{p(a,\{a, b, x\})}{p(x,\{a, b, x\})}$ and $\frac{p(b,\{b, x\})}{p(x,\{b, x\})}=\frac{p(b,\{a, b, x\})}{p(x,\{a, b, x\})}$. Hence, by Axiom $8, \frac{p(a, A)}{p(b, A)}=\frac{p(a, A \cup x)}{p(b, A \cup x)}$. Suppose $a \chi_{p} x$ and $b \chi_{p} x$. Then there are $B, C$ such that $\frac{p(a,\{a, x\})}{p(x,\{a, x\})} \neq \frac{p(a, B)}{p(x, B)}$ and $\frac{p(b,\{b, x\})}{p(x,\{b, x\})} \neq \frac{p(b, C)}{p(x, C)}$. Hence, by Axiom 9, $\frac{p(a, A)}{p(b, A)}=\frac{p(a, A \cup x)}{p(b, A \cup x)}$. Therefore, ISA is satisfied. Hence, by Theorem 1, $p$ is a nondegenerate NSC with some $\left(v, u,\left\{X_{i}\right\}_{i=1}^{K}\right)$ where $X / \sim_{p}=\left\{X_{i}\right\}_{i=1}^{K}$.

To show the strictness, take any $a, x \in X_{i}$ such that $\frac{u(a)+u(x)}{u(x)}=\frac{v(\{a, x\})}{v(x)}$. Then for any $b \in X_{j}$, $\frac{p(b,\{b, x\})}{p(x,\{b, x\})}=\frac{p(b,\{a, b, x\})}{p(x,\{a, b, x\})}$. Since $\frac{p(a,\{a, x\})}{p(x,\{a, x\})}=\frac{p(a,\{a, b, x\})}{p(x,\{a, b, x\})}$, by Axiom 8, we have $\frac{p(a, A)}{p(b, A)}=\frac{p(a, A \cup x)}{p(b, A \cup x)}$. By NSC, we have

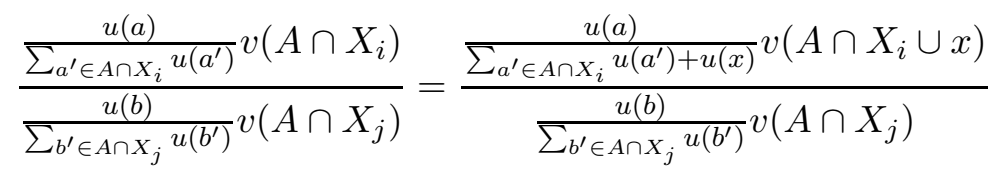

equivalently,

$$
\frac{\sum_{a^{\prime} \in A \cap X_{i}} u\left(a^{\prime}\right)+u(x)}{\sum_{a^{\prime} \in A \cap X_{i}} u\left(a^{\prime}\right)}=\frac{v\left(A \cap X_{i} \cup x\right)}{v\left(A \cap X_{i}\right)} .
$$

Necessity. Suppose $p$ is the strict nondegenerate NSC with $\left(v, u,\left\{X_{i}\right\}_{i=1}^{K}\right)$. By the necessity part of Theorem 1, $p$ satisfies ISA and $a \sim_{p} b$ if and only if $a, b \in X_{i}$.

To prove Axiom 8, take any $A \in \mathscr{A}, a, b \in A$, and $x \notin A$ with $\frac{p(a,\{a, x\})}{p(x,\{a, x\})}=\frac{p(a,\{a, b, x\})}{p(x,\{a, b, x\})}$ and $\frac{p(b,\{b, x\})}{p(x,\{b, x\})}=\frac{p(b,\{a, b, x\})}{p(x,\{a, b, x\})}$. We shall prove that $\frac{p(a, A)}{p(b, A)}=\frac{p(a, A \cup x)}{p(b, A \cup x)}$. It is immediate when $a, b \in X_{i}$. Hence, suppose $a \in X_{i}$ and $b \in X_{j}$. If $x \in X_{k}$, then we have $a \varkappa_{p} x$ and $b \varkappa_{p} x$. Consequently, by ISA, we have $\frac{p(b,\{b, x\})}{p(x,\{b, x\})}=\frac{p(b,\{a, b, x\})}{p(x,\{a, b, x\})}$. Hence, suppose now either $x \in X_{i}$ or $x \in X_{j}$. Since the role of $a$ and $b$ are symmetric, suppose $a \in X_{i}$ without loss of generality. By the NSC, $\frac{p(b,\{b, x\})}{p(x,\{b, x\})}=\frac{p(b,\{a, b, x\})}{p(x,\{a, b, x\})}$ implies $\frac{v(b)}{v(x)}=\frac{v(b)}{\frac{u(x)}{u(a)+u(x)} v(\{a, x\})}$; equivalently, $\frac{u(a)+u(x)}{u(x)}=\frac{v(\{a, x\})}{v(x)}$. Since by the 
strictness, $\frac{\sum_{a^{\prime} \in A_{i}} u\left(a^{\prime}\right)+u(x)}{\sum_{a^{\prime} \in A_{i}} u\left(a^{\prime}\right)}=\frac{v\left(A_{i} \cup x\right)}{v\left(A_{i}\right)}$ for any $A_{i} \subset X_{i}$.

By the NSC, $\frac{p(a, A)}{p(b, A)}=\frac{p(a, A \cup x)}{p(b, A \cup x)}$ is equivalent to

$$
\frac{\frac{u(a)}{\sum_{a^{\prime} \in A \cap X_{i}} u\left(a^{\prime}\right)} v\left(A \cap X_{i}\right)}{\frac{u(b)}{\sum_{b^{\prime} \in A \cap X_{j}} u\left(b^{\prime}\right)} v\left(A \cap X_{j}\right)}=\frac{\frac{u(a)}{\sum_{a^{\prime} \in A \cap X_{i}}^{u\left(a^{\prime}\right)+u(x)} v\left(A \cap X_{i} \cup x\right)}}{\frac{u(b)}{\sum_{b^{\prime} \in A \cap X_{j}} u\left(b^{\prime}\right)} v\left(A \cap X_{j}\right)} .
$$

The above equality holds by the strictness since it is equivalent to

$$
\frac{\sum_{a^{\prime} \in A \cap X_{i}} u\left(a^{\prime}\right)+u(x)}{\sum_{a^{\prime} \in A \cap X_{i}} u\left(a^{\prime}\right)}=\frac{v\left(A \cap X_{i} \cup x\right)}{v\left(A \cap X_{i}\right)} .
$$

To prove Axiom 9, take any $A, B, C \in \mathscr{A}, a \in A \cap B, b \in A \cap C$, and $x \in B \cap C$ with $\frac{p(a,\{a, x\})}{p(x,\{a, x\})} \neq \frac{p(a, B)}{p(x, B)}$ and $\frac{p(b,\{b, x\})}{p(x,\{b, x\})} \neq \frac{p(b, C)}{p(x, C)}$. Then $a \nsim_{p} x$ and $b \nsim_{p} x$. Hence, by ISA, $\frac{p(a, A)}{p(b, A)}=\frac{p(a, A \cup x)}{p(b, A \cup x)}$.

\section{B.4.4 Proof of Theorem 6}

We prove Theorem 6 by four steps.

Step 1. Note that the first part of Generalized Independence of Symmetric Alternatives is identical to the first part of Independence of Symmetric Alternatives. Hence, by Steps 1-2 of the proof of Theorem $1, \sim_{p}$ is reflexive, transitive, and symmetric, we have a partition $X / \sim_{p} \equiv\left\{E_{i}\right\}_{i=1}^{K}$ of $X$ such that for any $x_{i}, x_{i}^{\prime} \in E_{i}$ and $x_{j} \in E_{j}, x_{i} \sim_{p} x_{i}^{\prime}$ and $x_{i} \varkappa_{p} x_{j}$.

Step 2. $\simeq_{p}$ is transitive.

Take any $x, y, z \in X$ such that $x \simeq_{p} y$ and $y \simeq_{p} z$. If $x \sim_{p} y$ and $y \sim_{p} z$, then by Step 1 , $x \sim_{p} z$. If $x \sim_{p} y$ and $y \bowtie_{p} z$, then by Consistency of Revealed Similarities, $x \bowtie_{p} z$. If $x \bowtie_{p} y$ and $y \sim_{p} z$, then by Consistency of Revealed Similarities, $x \bowtie_{p} z$. Finally, we consider the case where $x \bowtie_{p} y$ and $y \bowtie_{p} z$.

Suppose $x \not_{p} z$. Then we shall prove that $x \bowtie_{p} z$; i.e., for any $A$ and $t \notin A$ such that $x \varkappa_{p} t$ and $z \chi_{p} t, \frac{p(x, A)}{p(z, A)}=\frac{p(x, A \cup t)}{p(z, A \cup t)}$.

Case 1. $y \in A$. In this case, we can write $\frac{p(x, A)}{p(z, A)}=\frac{p(x, A)}{p(y, A)} / \frac{p(z, A)}{p(y, A)}$ and $\frac{p(x, A \cup t)}{p(z, A \cup t)}=\frac{p(x, A \cup t)}{p(y, A \cup t)} / \frac{p(z, A \cup t)}{p(y, A \cup t)}$. If $y \varkappa_{p} t, x \bowtie_{p} y$ implies $\frac{p(x, A)}{p(y, A)}=\frac{p(x, A \cup t)}{p(y, A \cup t)}$ and $y \bowtie_{p} z$ implies $\frac{p(z, A)}{p(y, A)}=\frac{p(z, A \cup t)}{p(y, A \cup t)}$. Therefore, $\frac{p(x, A)}{p(z, A)}=\frac{p(x, A)}{p(y, A)} / \frac{p(z, A)}{p(y, A)}=\frac{p(x, A \cup t)}{p(z, A \cup t)}=\frac{p(x, A \cup t)}{p(y, A \cup t)} / \frac{p(z, A \cup t)}{p(y, A \cup t)}$. Instead, if $y \sim_{p} t$, then by Consistency of Revealed Preferences $x \bowtie_{p} y$ and $y \bowtie_{p} z$ imply $x \bowtie_{p} t$ and $z \bowtie_{p} t$. Then by Generalized Independence of Symmetric Alternatives, $\frac{p(x, A)}{p(z, A)}=\frac{p(x, A \cup t)}{p(z, A \cup t)}$.

Case 2. $y \notin A$. By Generalized Independence of Symmetric Alternatives, $x \bowtie_{p} y$ and $z \bowtie_{p} y$ imply $\frac{p(x, A)}{p(z, A)}=\frac{p(x, A \cup y)}{p(z, A \cup y)}$ and $\frac{p(x, A \cup t)}{p(z, A \cup t)}=\frac{p(x, A \cup y \cup t)}{p(z, A \cup y \cup t)}$. Now by Case $1, \frac{p(x, A)}{p(z, A)}=\frac{p(x, A \cup y)}{p(z, A \cup y)}=\frac{p(x, A \cup y \cup t)}{p(z, A \cup y \cup t)}=$ $\frac{p(x, A \cup t)}{p(z, A \cup t)}$.

Step 3. Let $X / \simeq_{p} \equiv\left\{X_{i}\right\}_{i=1}^{n}$. Since $\simeq_{p}$ is reflexive, transitive, and symmetric, $\left\{X_{i}\right\}_{i=1}^{n}$ is a partition of $X$ such that for any $x_{i}, x_{i}^{\prime} \in X_{i}$ and $x_{j} \in X_{j}, x_{i} \simeq_{p} x_{i}^{\prime}$ and $x_{i} \varkappa_{p} x_{j}$. Moreover, by the definition of $\simeq_{p}$, for any $i \leq K$, there is some $j \leq n$ such that $E_{i} \subseteq X_{j}$. Hence, without loss of 
generality, let $X_{i}=\bigcup_{s=1}^{t_{i}} X_{i}^{s}$ such that for any $s \leq t_{i}, X_{i}^{s}=E_{l}$ for some $l \leq K$.

Step 4. For any $x, y \in X_{i}, x \bowtie_{p} y$ if and only if $x \nsim y$. Hence, the first two parts of Generalized Independence of Symmetric Alternatives are equivalent to Independence of Symmetric Alternatives when $p$ is restricted on $X_{i}$. Hence, by Theorem $1, p$ is an NSC on $X_{i}$ with some $\left(u_{i}, v_{i},\left\{X_{i}^{s}\right\}_{s=1}^{t_{i}}\right)$. Since $X_{i}$ and $X_{j}$ are disjoint for each $i, j$ with $j \neq i$, without loss of generality, we can say that $p$ is an NSC on $X_{i}$ with the same $(v, u)$.

Step 5. Since there are $a, b, c \in X$ such that $a \neq_{p} b, b \neq_{p} c$, and $a 千_{p} c$, we have $n \geq 3$. Take any $A \in \mathscr{A}$. Let $A_{i}=A \cap X_{i}$ for each $i \leq n$. Take any $a \in A_{i}, b \in A_{j}$, and $x \in A_{k}$. Note that $a \neq_{p} x$ and $b 千_{p} x$. Then by Generalized Independence of Symmetric Alternatives, we have

$$
\frac{p(a, A)}{p(b, A)}=\frac{p(a, A \backslash\{x\})}{p(b, A \backslash\{x\})} .
$$

Then by Steps 5-8 of Theorem 1 (also recall Equation (12)), there is a function $w: 2^{X} \rightarrow \mathbb{R}_{+}$such that $\frac{p\left(A_{i}, A\right)}{p\left(A_{j}, A\right)}=\frac{w\left(A_{i}\right)}{w\left(A_{j}\right)}$. In other words, $p\left(A_{i}, A\right)=\frac{w\left(A_{i}\right)}{\sum_{j} w\left(A_{j}\right)}$. Since $p$ is an NSC on $X_{i}$, we also have

$$
p\left(a, A_{i}\right)=\frac{v\left(A_{i} \cap X_{i}^{s}\right)}{\sum_{l} v\left(A_{i} \cap X_{i}^{l}\right)} \frac{u(a)}{\sum_{b \in A_{i} \cap X_{i}^{s}} u(b)}
$$

when $a \in A_{i} \cap X_{i}^{s}$. Finally,

$$
p(a, A)=p\left(A_{i}, A\right) p\left(a, A_{i}\right)=\frac{w\left(A_{i}\right)}{\sum_{j} w\left(A_{j}\right)} \frac{v\left(A_{i} \cap X_{i}^{s}\right)}{\sum_{l} v\left(A_{i} \cap X_{i}^{l}\right)} \frac{u(a)}{\sum_{b \in A_{i} \cap X_{i}^{s}} u(b)} .
$$

